TRANSACTIONS OF THE

AMERICAN MATHEMATICAL SOCIETY

Volume 364, Number 6, June 2012, Pages 3185-3216

S 0002-9947(2012)05476-6

Article electronically published on February 8, 2012

\title{
DIRAC OPERATORS ON COBORDISMS: DEGENERATIONS AND SURGERY
}

\author{
DANIEL F. CIBOTARU AND LIVIU I. NICOLAESCU
}

\begin{abstract}
We investigate the Dolbeault operator on a pair of pants, i.e., an elementary cobordism between a circle and the disjoint union of two circles. This operator induces a canonical selfadjoint Dirac operator $D_{t}$ on each regular level set $C_{t}$ of a fixed Morse function defining this cobordism. We show that as we approach the critical level set $C_{0}$ from above and from below these operators converge in the gap topology to (different) selfadjoint operators $D_{ \pm}$ that we describe explicitly. We also relate the Atiyah-Patodi-Singer index of the Dolbeault operator on the cobordism to the spectral flows of the operators $D_{t}$ on the complement of $C_{0}$ and the Kashiwara-Wall index of a triplet of finite dimensional Lagrangian spaces canonically determined by $C_{0}$.
\end{abstract}

\section{INTRODUCTION}

Suppose $(M, g)$ is a compact oriented odd-dimensional Riemann manifold. We let $\widehat{M}$ denote the cylinder $[0,1] \times M$ and $\hat{g}$ denote the cylindrical metric $d t^{2}+g$.

Let $\hat{D}$ be a first order elliptic operator on a vector bundle over $\widehat{M}$ that has the form

$$
\widehat{D}=\sigma(d t)\left(\nabla_{t}-D(t)\right),
$$

where $\sigma$ denotes the principal symbol of $\widehat{D}$, and for every $t \in[0,1]$ the operator $D(t)$ on $\{t\} \times M$ is elliptic and symmetric. For simplicity we assume that both $D(0)$ and $D(1)$ are invertible.

A classical result of Atiyah, Patodi and Singer [2, §7] (see also [12, §17.1]) relates the index $i_{A P S}(\widehat{D})$ of the Atiyah-Patodi-Singer problem associated to $\widehat{D}$ to the spectral flow $S F(D(t))$ of the family of Fredholm selfadjoint operators $D(t)$. More precisely, they show that

$$
i_{A P S}(\widehat{D})+S F(D(t), 0 \leq t \leq 1)=0 .
$$

We can regard the cylinder $\widehat{M}$ as a trivial cobordism between $\{0\} \times M$ and $\{1\} \times M$, and the coordinate $t$ as a Morse function on $\widehat{M}$ with no critical points.

In this paper we initiate an investigation of the case when $\widehat{M}$ is no longer a trivial cobordism. We outline below the main themes of this investigation.

First, we will concentrate only on elementary cobordisms, the ones that trace a single surgery. We regard such a cobordism as a pair $(\widehat{M}, f)$, where $\widehat{M}$ is an evendimensional, compact oriented manifold with boundary, and $f$ is a Morse function

Received by the editors February 9, 2010 and, in revised form, September 22, 2010.

2010 Mathematics Subject Classification. Primary 58J20, 58J28, 58J30, 58J32, 53B20, 35B25.

The second author was partially supported by NSF grant DMS-1005745.

(C)2012 American Mathematical Society Reverts to public domain 28 years from publication 
on $\widehat{M}$ with a single critical point $p_{0}$ such that

$$
f(\widehat{M})=[-1,1], \quad f(\partial M)=\{-1,1\}, \quad f\left(p_{0}\right)=0 .
$$

We set $M_{ \pm}:=f^{-1}( \pm 1)$ so that we have a diffeomorphism of oriented manifolds $\partial M=M_{+} \cup-M_{-}$. Suppose that $\hat{g}$ is a Riemann metric on $\widehat{M}$ and $\widehat{D}: C^{\infty}\left(E_{+}\right) \rightarrow$ $C^{\infty}\left(E_{-}\right)$is a Dirac type operator on $\widehat{M}$, where $E_{+} \oplus E_{-}$is a $\mathbb{Z} / 2$-graded bundle of Clifford modules.

Using the unitary bundle isomorphism $\frac{1}{|d f|} \sigma(d f): E_{+} \rightarrow E_{-}$defined away from the critical level set, we can regard $\left.\widehat{D}\right|_{\{f \neq 0\}}$ as an operator $C^{\infty}\left(E_{+}\right) \rightarrow C^{\infty}\left(E_{+}\right)$. As explained in [8] (see also Section 2 of this paper), for every $t \neq 0$, there is a canonically induced symmetric Dirac operator $D(t)$ on the slice $M_{t}=f^{-1}(t)$. We regard $D(t)$ as a linear operator $D(t): C^{\infty}\left(\left.E_{+}\right|_{M_{t}}\right) \rightarrow C^{\infty}\left(\left.E_{+}\right|_{M_{t}}\right)$, so that, if $\hat{g}$ were a cylindrical metric, then formula (1T) would hold.

The Riemann metric $\hat{g}$ defines finite measures $d V_{t}$ on all the slices $M_{t}$, including the singular slice $M_{0}$. In particular, we obtain a one-parameter family of Hilbert spaces

$$
\boldsymbol{H}_{t}:=L^{2}\left(M_{t}, d V_{t} ; E_{+}\right) .
$$

We can now regard $D(t)$ as a closed, densely defined linear operator on $\boldsymbol{H}_{t}$.

Problem 1. Organize the family $\left(\boldsymbol{H}_{t}\right)_{t \in[-1,1]}$ as a trivial Hilbert bundle over the interval $[-1,1]$,

$$
\mathcal{H}=\boldsymbol{H} \times[-1,1] \rightarrow[-1,1]
$$

Under reasonable assumptions on $f$ and $\hat{g}$ we can use the gradient flow of $f$ to address this issue. Once this problem is solved we can regard the operators $D(t)$, $t \neq 0$, as closed densely defined operators on the same Hilbert space $\boldsymbol{H}$. We can then formulate our next problem.

Problem 2. Investigate whether the limits

$$
S F_{-}:=\lim _{\varepsilon \searrow 0} S F(D(t),-1 \leq t \leq-\varepsilon), \quad S F_{+}:=\lim _{\varepsilon \searrow 0} S F(D(t), \varepsilon \leq t \leq 1)
$$

exist and are finite.

If Problem 2 has a positive answer we are interested in a version of (A) relating these limits to the Atiyah-Patodi-Singer index of $\widehat{D}$ in the noncylindrical formulation of $[8,9]$.

Problem 3. Express the quantity

$$
\delta:=i_{A P S}(\widehat{D})+S F_{-}+S F_{+}
$$

in terms of invariants of the singular level set $M_{0}$.

The existence of the limits in Problem 2 is a consequence of a much more refined analytic behavior of the family of operators $D(t)$ that we now proceed to explain. We set

$$
\widehat{\boldsymbol{H}}:=\boldsymbol{H} \oplus \boldsymbol{H}, \quad \boldsymbol{H}_{+}:=\boldsymbol{H} \oplus 0, \quad \boldsymbol{H}_{-}:=0 \oplus \boldsymbol{H},
$$

and we denote by $\boldsymbol{L a g}$ the Grassmannian of Hermitian Lagrangian subspaces $\widehat{\boldsymbol{H}}$. These are complex subspaces $L \subset \widehat{\boldsymbol{H}}$ satisfying $L^{\perp}=J L$, where $J: \boldsymbol{H} \oplus \boldsymbol{H} \rightarrow$ $\boldsymbol{H} \oplus \boldsymbol{H}$ is the operator with block decomposition

$$
J=\left[\begin{array}{cc}
0 & -1 \\
1 & 0
\end{array}\right] .
$$


Following [5] we denote by $\mathbf{L a g}^{-}$the open subset of $\mathbf{L a g}$ consisting of Lagrangians $L$ such that the pair of subspaces $\left(L, \widehat{\boldsymbol{H}}_{-}\right)$is a Fredholm pair, i.e.,

$$
L+\boldsymbol{H}_{-} \text {is closed and } \operatorname{dim} L \cap \boldsymbol{H}_{-}<\infty .
$$

As explained in [5], the space $\mathbf{L a g}^{-}$equipped with the gap topology of [10, §IV.2] is a classifying space for the complex $K$-theoretic functor $K^{1}$.

To a closed densely defined operator $T: \operatorname{Dom}(T) \subset \boldsymbol{H} \rightarrow \boldsymbol{H}$ we associate its switched graph

$$
\widetilde{\Gamma}_{T}:=\{(T h, h) \in \widehat{\boldsymbol{H}} ; h \in \operatorname{Dom}(T)\} .
$$

Then $T$ is selfadjoint if and only if $\widetilde{\Gamma}_{T} \in \boldsymbol{L a g}$. It is also Fredholm if and only if $\widetilde{\Gamma}_{T} \in \mathbf{L a g}^{-}$. We can now formulate a refinement of Problem 2.

Problem $2 *$. Investigate whether the limits $\widetilde{\Gamma}_{ \pm}=\lim _{t \searrow 0} \widetilde{\Gamma}_{D( \pm t)}$ exist in the gap topology and, if so, do they belong to $\mathbf{L a g}^{-}$.

The gap convergence of the switched graphs of operators is equivalent to the convergence in norm as $t \rightarrow 0^{ \pm}$of the (compact) resolvents $R_{t}=(i+D(t))^{-1}$. To show that $\widetilde{\Gamma}_{ \pm} \in \mathbf{L a g}^{-}$it suffices to show that the limits $R_{ \pm}=\lim _{t \rightarrow 0^{ \pm}} R_{t}$ exist. Automatically, these limits will be compact operators, which guarantees that the limits belong to $\boldsymbol{L a g}^{-}$. If in addition $11 \widetilde{\Gamma}_{ \pm} \cap \widehat{\boldsymbol{H}}_{-}=0$, then the limits in Problem 2 exist and are finite.

An even analog of Problem $2^{*}$ was investigated in [16. The role of the smooth slices $M_{t}$ was played there by a 1-parameter family of Riemann surfaces degenerating to a Riemann surface with single singularity of the simplest type, a node. The authors show that the gap limit of the graphs of Dolbeault operators on $M_{t}$ exists and they described it explicitly.

In this paper we solve Problems $1,2^{*}$ and 3 in the simplest possible case, when $\widehat{M}$ is an elementary 2-dimensional cobordism, i.e., a pair of pants (see Figure 1) and $\widehat{D}$ is the Dolbeault operator on the Riemann surface $\widehat{M}$. The other possibility, namely the cobordism corresponding to the case when the critical point is a local minimum/maximum, is not very complicated, but it displays an interesting analytical phenomenon. We discuss it at length in Remark 3.3

We solved Problem 1 by an ad-hoc intuitive method. The limits $\widetilde{\Gamma}_{ \pm}$in Problem $2^{*}$ turned out to be switched graphs of certain Fredholm selfadjoint operators $D_{ \pm}$, $\widetilde{\Gamma}_{ \pm}=\widetilde{\Gamma}_{D_{ \pm}}$.

We describe these limiting operators as realizations of two different boundary value problems associated to the same symmetric Dirac operator $D_{0}$ defined on the disjoint union of four intervals. These intervals are obtained by removing the singular point of the critical level set $M_{0}$ and then cutting in half each of the resulting two components. The boundary conditions defining $D_{ \pm}$are described by some (4-dimensional) Hermitian Lagrangians $\Lambda_{ \pm}$determined by the geometry of the singular slice $M_{0}$. The operators $D_{ \pm}$have well-defined eta invariants $\eta_{ \pm}$. If ker $D_{ \pm}=0$, then we can express the defect $\delta$ in (B) as

$$
\delta=\frac{1}{2}\left(\eta_{-}-\eta_{+}\right) .
$$

\footnotetext{
${ }^{1}$ The condition $\widetilde{\Gamma}_{ \pm} \cap \widehat{\boldsymbol{H}}_{-}=0$ is not really needed, but it makes our presentation more transparent. In any case, it is generically satisfied.
} 
The above difference of eta invariants admits a purely symplectic interpretation very similar to the signature additivity defect of Wall [19]. More precisely, we show that

$$
\delta=-\omega\left(\Lambda_{0}^{\perp}, \Lambda_{+}, \Lambda_{-}\right)
$$

where $\Lambda_{0}$ is the Cauchy data space of the operator $D_{0}$ and $\omega\left(L_{0}, L_{1}, L_{2}\right)$ denotes the Kashiwara-Wall index of a triplet of Lagrangians canonically determined by $M_{0}$; see [4, 11, 19] or Section 4,

Here is briefly how we structured the paper. In Section 1 we investigate in great detail the type of degenerations that occur in the family $D(t)$ as $t \rightarrow 0^{ \pm}$. It boils down to understanding the behavior of families of operators of the unit circle $S^{1}$ of the type

$$
L_{\varepsilon}=-i \frac{d}{d \theta}+a_{\varepsilon}(\theta)
$$

where $\left\{a_{\varepsilon}\right\}_{\varepsilon>0}$ is a family of smooth functions on the unit circle that converges in a rather weak sense way as $\varepsilon \rightarrow 0$ to a Dirac measure supported at a point $\theta_{0}$. For example, if we think of $a_{\varepsilon}$ as densities defining measures converging weakly to the Dirac measure, then the corresponding family of operators has a well-defined gap limit; see Corollary 1.5 .

In Theorem 1.8 we give an explicit description of this limiting operator as an operator realizing a natural boundary value problem on the disjoint union of the two intervals, $\left[0, \theta_{0}\right]$ and $\left[\theta_{0}, 2 \pi\right]$. The boundary conditions have natural symplectic interpretations. This section also contains a detailed discussion of the eta invariants of operators of the type $-\boldsymbol{i} \frac{d}{d \theta}+a(\theta)$, where $a$ is allowed to be the "density" of any finite Radon measure.

In Section 2 we survey mostly known facts concerning the Atiyah-Patodi-Singer problem when the metric near the boundary is not cylindrical. Because the various orientation conventions vary wildly in the existing literature, we decided to go carefully through the computational details. We discuss two topics. First, we explain what is the restriction of a Dirac operator to a cooriented hypersurface and relate this construction to another conceivable notion of restriction. In the second part of this section we discuss the noncylindrical version of the Atiyah-Patodi-Singer index theorem. Here we follow closely the presentation in [8, 9].

In Section 3 we formulate and prove the main result of this paper, Theorem 3.1 . The solution to Problem $2^{*}$ is obtained by reducing the study of the degenerations to the model degenerations investigated in Section 1 . The equality (C) follows immediately from the noncyclindrical version of the Atiyah-Patodi-Singer index theorem discussed in Section 2 and the eta invariant computations in Section 1 In the last section we present a few facts about the Kashiwara-Wall triple index and then use them to prove (D). Our definition of a triple index is the one used by Kirk and Lesch [1] that generalizes to infinite dimensions.

Let us say a few words about conventions and notation: We consistently orient the boundaries using the outer-normal-first convention. We let $i$ stand for $\sqrt{-1}$ and we let $L^{k, p}$ denote Sobolev spaces of functions that have weak derivatives up to order $k$ that belong to $L^{p}$. 


\section{A MODEL DEGENERATION}

Let $L>0$ be a positive number. Denote by $\boldsymbol{H}$ the Hilbert space $L^{2}([0, L], \mathbb{C})$. To any measurable function $a: \mathbb{R} \rightarrow \mathbb{R}$ which is bounded 2 and $L$-periodic we associate the selfadjoint operator

$$
D_{a}: \operatorname{Dom}\left(D_{a}\right) \subset \boldsymbol{H} \rightarrow \boldsymbol{H}
$$

where

$$
\operatorname{Dom}\left(D_{a}\right)=\left\{u \in L^{1,2}([0, L], \mathbb{C}) ; u(0)=u(L)\right\}, \quad D_{a} u=-i \frac{d u}{d t}+a u .
$$

In this section we would like to understand the dependence of $D_{a}$ on the potential $a$, and in particular, we would like to allow for more singular potentials such as a Dirac distribution concentrated at an interior point of the interval. We will reach this goal via a limiting procedure that we implement in several steps.

We observe first that $D_{a}$ can be expressed in terms of the resolvent $R_{a}:=$ $\left(\boldsymbol{i}+D_{a}\right)^{-1}$ as $D_{a}=R_{a}^{-1}-\boldsymbol{i}$. The advantage of this point of view is that we can express $R_{a}$ in terms of the more regular function

$$
A(t):=\int_{0}^{t} a(s) d s
$$

which continues to make sense even when there is no integrable function $a$ such

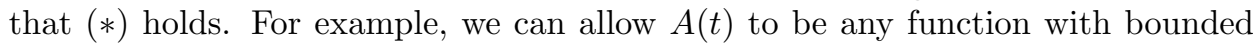
variation so that, formally, a ought to be the density of any Radon measure on $[0, L]$.

This will allow us to conclude that when we have a family of smooth potentials $a_{n}$ that converge in a suitable sense to something singular such as a Dirac function, then the operators $D_{a_{n}}$ have a limit in the gap topology to a Fredholm selfadjoint operator with compact resolvent. We show that in many cases this limit operator can be expressed as the Fredholm operator defined by a boundary value problem.

We begin by expressing $R_{a}$ as an integral operator. We set

$$
A(t):=\int_{0}^{t} a(s) d s, \quad \Phi_{A}(t):=\boldsymbol{i} A(t)-t
$$

For $f \in H$ the function $u=R_{a} f$ is the solution of the boundary value problem

$$
\left(\boldsymbol{i}-\boldsymbol{i} \frac{d}{d t}\right) u+a u=f, u(0)=u(L) .
$$

An elementary computation yields the equality

$$
u(t)=R_{a} f=\frac{\boldsymbol{i} e^{-\Phi_{A}(t)}}{e^{\Phi_{A}(L)}-1} \int_{0}^{L} e^{\Phi_{A}(s)} f(s) d s+\boldsymbol{i} \int_{0}^{t} e^{-\left(\Phi_{A}(t)-\Phi_{A}(s)\right)} f(s) d s .
$$

The key point of the above formula is that $R_{a}$ can be expressed in terms of the antiderivative $A(t)$ which typically has milder singularities than $a$. To analyze the dependence of $R_{a}$ on $A$ we introduce a class of admissible functions.

Definition 1.1. (a) We say that $A:[0, L] \rightarrow \mathbb{R}$ is admissible if $A$ has bounded variation, it is right continuous, and $A(0)=0$. We denote by $\mathcal{A}$ or $\mathcal{A}_{L}$ the class of admissible functions.

\footnotetext{
${ }^{2}$ The assumption $a \in L^{\infty}$ guarantees that: 1) $\left.a u \in L^{2}(0, L), \forall u \in L^{1,2}(0, L) ; 2\right)$ the densely defined operator $D_{a}$ is closed.
} 
(b) We say that a sequence $\left\{A_{n}\right\}_{n \geq 0} \subset \mathcal{A}$ converges very weakly to $A \in \mathcal{A}$ if there exists a null measure subset $\Delta \subset(0, L)$ such that

$$
\lim _{n \rightarrow \infty} A_{n}(t)=A(t), \quad \forall t \in[0, L] \backslash \Delta .
$$

Remark 1.2. (a) Note that if $A_{n}$ converges very weakly to $A$, then $A_{n}(L)$ converges to $A(L)$.

(b) Let us explain the motivation behind the "very weak" terminology. An admissible function $A$ defines a finite Lebesgue-Stieltjes measure $\mu_{A}$ on $[0, L]$, and the resulting map $A \mapsto \mu_{A}$ is a linear isomorphism between $\mathcal{A}$ and the space of finite Borel measures on $[0, L], 7$, Thm. 3.29]. Thus, we can identify $\mathcal{A}$ with the space of finite Borel measures on $[0, L]$. As such it is equipped with a weak topology.

According to [6, §4.22], a sequence of Borel measures $\mu_{A_{n}}$ is weakly convergent to $\mu_{A}$ if and only if $\mu_{A_{n}}(\mathcal{O}) \rightarrow \mu_{A}(\mathcal{O})$, for any (relatively) open subset $\mathcal{O}$ of $[0, L]$. This clearly implies the very weak convergence introduced in Definition 1.1

Inspired by (1.2), we define for every $A \in \mathcal{A}$ the function $\Phi_{A}(t)=\boldsymbol{i} A(t)-t$ and the integral kernels

$$
\begin{aligned}
& \mathcal{S}_{A}:[0, L] \times[0, L] \rightarrow \mathbb{C}, \quad \mathcal{S}_{A}(t, s)=\frac{i}{e^{\Phi_{A}(L)}-1} e^{-\left(\Phi_{A}(t)-\Phi_{A}(s)\right)}, \quad \forall t, s \in[0, L], \\
& \mathcal{K}_{A}:[0, L] \times[0, L] \rightarrow \mathbb{C}, \mathcal{K}_{A}(t, s)= \begin{cases}0 & t<s, \\
\boldsymbol{i} e^{-\left(\Phi_{A}(t)-\Phi_{A}(s)\right)} & t \geq s .\end{cases}
\end{aligned}
$$

Observe that there exists a constant $C>0$ such that

$$
\left\|\mathcal{S}_{A}\right\|_{L^{\infty}([0, L] \times[0, L])}+\left\|\mathcal{K}_{A}\right\|_{L^{\infty}([0, L] \times[0, L])} \leq C, \quad \forall A \in \mathcal{A} .
$$

Thus, these kernels define bounded compact operators $S_{A}, K_{A}: \boldsymbol{H} \rightarrow \boldsymbol{H}$; see [18, $\S X .2]$. Moreover, if we denote by $\|\bullet\|_{\text {op }}$ the operator norm on the space $\mathcal{B}(\boldsymbol{H})$ of bounded linear operators $\boldsymbol{H} \rightarrow \boldsymbol{H}$, then we have the estimates

$$
\left\|S_{A}\right\|_{\mathrm{op}} \leq\left\|\mathcal{S}_{A}\right\|_{L^{2}([0, L] \times[0, L])}, \quad\left\|K_{A}\right\|_{\mathrm{op}} \leq\left\|\mathcal{K}_{A}\right\|_{L^{2}([0, L] \times[0, L])} .
$$

We can now rewrite (1.2) as

$$
R_{a}=R_{A}:=S_{A}+K_{A} .
$$

Proposition 1.3. If $A_{n}$ converges very weakly to $A$, then $S_{A_{n}}$ and $K_{A_{n}}$ converge in the operator norm topology to $S_{A}$ and respectively $K_{A}$.

Proof. The very weak convergence implies that

$$
\mathcal{S}_{A_{n}}(t, s) \stackrel{k \rightarrow \infty}{\longrightarrow} \mathcal{S}_{A}(t, s), \mathcal{K}_{A_{n}}(t, s) \stackrel{k \rightarrow \infty}{\longrightarrow} \mathcal{K}_{A}(t, s) \text { a.e. on }[0, L] \times[0, L] .
$$

Using (1.3), the above pointwise convergence and the dominated convergence theorem we deduce

$$
\lim _{n \rightarrow \infty}\left(\left\|\mathcal{S}_{A_{n}}-\mathcal{S}_{A}\right\|_{L^{2}([0, L] \times[0, L])}+\left\|\mathcal{K}_{A_{n}}-\mathcal{K}_{A}\right\|_{L^{2}([0, L] \times[0, L])}\right)=0 .
$$

The inequalities (1.4) now imply that

$$
\lim _{n \rightarrow \infty}\left(\left\|S_{A_{n}}-S_{A}\right\|_{\mathrm{op}}+\left\|S_{A_{n}}-S_{A}\right\|_{\mathrm{op}}\right)=0 .
$$


We want to describe the spectral decompositions of the operators $R_{A}, A \in \mathcal{A}$. To do this we rely on the fact that, for certain $A$ 's, the operator $R_{A}$ is the resolvent of an elliptic selfadjoint operator on $S^{1}$. We use this to produce an intelligent guess for the spectrum of $R_{A}$ in general.

Let $a$ be a smooth, real-valued, $L$-period function on $\mathbb{R}$ and form again the operator $D_{a}$ defined in (1.1). We set as usual

$$
A(t):=\int_{0}^{t} a(s) d s
$$

The operator $D_{a}$ has discrete real spectrum. If $u(t)$ is an eigenfunction corresponding to an eigenvalue $\lambda$, then

$$
-i \frac{d u}{d t}+a u=\lambda u \Rightarrow \frac{d u}{d t}+i(a-\lambda) u=0
$$

so that $u(t)=u(0) e^{-\boldsymbol{i} A(t)+\boldsymbol{i} \lambda t}$. The periodicity assumption implies $\lambda L-A(L) \in$ $2 \pi \mathbb{Z}$, so the spectrum of $D_{a}$ is

$$
\operatorname{spec}\left(D_{a}\right)=\left\{\lambda_{A, n}:=\frac{2 \pi}{L}\left(\omega_{A}+n\right) ; n \in \mathbb{Z}\right\}, \text { where } \omega_{A}:=\frac{A(L)}{2 \pi} .
$$

The eigenvalue $\lambda_{A, n}$ is simple and the eigenspace corresponding to $\lambda_{A, n}$ is spanned by

$$
\psi_{A, n}(t):=e^{\frac{2 \pi n i t}{L}} e^{-i\left(A(t)-\frac{A(L) t}{L}\right)} .
$$

The numbers $\lambda_{A, n}$ and the functions $\psi_{A, n}$ are well defined for any $A \in \mathcal{A}$.

Lemma 1.4. Let $A \in \mathcal{A}$. Then the collection $\left\{\psi_{A, n}(t) ; n \in \mathbb{Z}\right\}$ defines a Hilbert basis of $\boldsymbol{H}$.

Proof. Observe first that the collection

$$
e_{n}(t)=\psi_{A=0, n}(t)=e^{\frac{2 \pi n i t}{L}}, n \in \mathbb{Z}
$$

is the canonical Hilbert basis of $\boldsymbol{H}$ that leads to the classical Fourier decomposition. The map

$$
U_{A}: \boldsymbol{H} \rightarrow \boldsymbol{H}, \quad \boldsymbol{H} \ni f(t) \mapsto e^{-i\left(A(t)-\frac{A(L) t}{L}\right)} f(t)
$$

is unitary. It maps $e_{n}$ to $\psi_{A, n}$, which proves our claim.

A direct computation shows that

$$
R_{A} \psi_{A, n}=\frac{1}{\boldsymbol{i}+\lambda_{A, n}} \psi_{A, n}, \quad \forall A \in \mathcal{A}, \quad A \in \mathcal{A} .
$$

This proves that for any $A \in \mathcal{A}$ the collection $\left\{\psi_{A, n}\right\}_{n \in \mathbb{Z}}$ is a Hilbert basis that diagonalizes the operator $R_{A}$. Observe that $R_{A}$ is injective and compact. We define

$$
T_{A}:=R_{A}^{-1}-i .
$$

The operator $T_{A}$ is unbounded, closed and densely defined with domain $\operatorname{Dom}\left(T_{A}\right)=$ Range $\left(R_{A}\right)$. Later we will present a more explicit description of $\operatorname{Dom}\left(T_{A}\right)$ for a large class of $A$ 's. Note that when

$$
A=\int_{0}^{t} a(s) d s, \quad a \text { smooth and } L \text {-periodic, }
$$

the operator $T_{A}$ coincides with the operator $D_{a}$ defined in (1.1). Proposition 1.3 can be rephrased as follows. 
Corollary 1.5. If the sequence $\left(A_{n}\right)_{n \geq 1} \subset \mathcal{A}$ converges very weakly to $A \in \mathcal{A}$, then the sequence of unbounded operators $\left(T_{A_{n}}\right)_{n \geq 1}$ converges in the gap topology to the unbounded operator $T_{A}$.

The spectrum of $T_{A}$ consists only of the simple eigenvalues $\lambda_{A, n}, n \in \mathbb{Z}$. The function $\psi_{A_{n}}$ is an eigenfunction of $T_{A}$ corresponding to the eigenvalue $\lambda_{A, n}$. The eta invariant of $T_{A}$ is now easy to compute. For $s \in \mathbb{C}$ we have

$$
\begin{aligned}
& \eta_{A}(s):=\sum_{\lambda>0} \frac{1}{\lambda^{s}}\left(\operatorname{dim} \operatorname{ker}\left(\lambda-T_{A}\right)-\operatorname{dim} \operatorname{ker}\left(\lambda+T_{A}\right)\right) \\
& =\sum_{n \in \mathbb{Z} \backslash\left\{-\omega_{A}\right\}} \frac{\operatorname{sign} \lambda_{A, n}}{\left|\lambda_{A, n}\right|^{s}}=\frac{L^{s}}{2 \pi^{s}} \sum_{n \in \mathbb{Z} \backslash\left\{-\omega_{A}\right\}} \frac{\operatorname{sign}\left(n+\omega_{A}\right)}{\left|n+\omega_{A}\right|^{s}} .
\end{aligned}
$$

Let

$$
\rho_{A}:=\omega_{A}-\left\lfloor\omega_{A}\right\rfloor=\frac{A(L)}{2 \pi}-\left\lfloor\frac{A(L)}{2 \pi}\right\rfloor \in[0,1) .
$$

If $\rho_{A}=0$, then $\eta_{A}(s)=0$ because in this case the spectrum of $T_{A}$ is symmetric about the origin. If $\rho_{A} \neq 0$, then we have

$\eta_{A}(s)=\frac{L^{s}}{2 \pi^{s}}\left(\sum_{n \geq 0} \frac{1}{\left(n+\rho_{A}\right)^{s}}-\sum_{n \geq 0} \frac{1}{\left(n+1-\rho_{A}\right)^{s}}\right)=\frac{L^{s}}{2 \pi^{s}}\left(\zeta\left(s, \rho_{A}\right)-\zeta\left(s, 1-\rho_{A}\right)\right)$,

where for every $\rho \in(0,1]$ we denoted by $\zeta(s, \rho)$ the Riemann-Hurwitz zeta function

$$
\zeta(s, \rho)=\sum_{n \geq 0} \frac{1}{(n+\rho)^{s}} .
$$

The above series is convergent for any $s \in \mathbb{C}, \boldsymbol{R e} s>1$, and admits an analytic continuation to the punctured plane $\mathbb{C} \backslash\{s=1\}$. Its value at the origin $s=0$ is given by Hermite's formula [17, §13.21]

$$
\zeta(0, \rho)=\frac{1}{2}-\rho
$$

We deduce that $\eta_{A}(s)$ has an analytic continuation at $s=0$ and we have

$$
\eta_{A}(0)=\left\{\begin{array}{cll}
0 & \text { if } & \rho_{A}=0, \\
1-2 \rho_{A} & \text { if } & \rho_{A} \in(0,1) .
\end{array}\right.
$$

Following [2, Eq.(3.1)], we introduce the reduced eta function

$$
\xi_{A}:=\frac{1}{2}\left(\operatorname{dim} \operatorname{ker} T_{A}+\eta_{A}(0)\right)
$$

Then we can rewrite the above equality in a more compact way:

$$
\xi_{A}=\frac{1}{2}\left(1-2 \rho_{A}\right)=\frac{1}{2}-\rho_{A} .
$$

Suppose we have $A_{0}, A_{1} \in \mathcal{A}$. We set $A_{s}=A_{0}+s\left(A_{1}-A_{0}\right) \in \mathcal{A}$. The map $[0,1] \ni s \mapsto A_{s} \in \mathcal{A}$ is continuous in the weak topology on $\mathcal{A}$ and thus the family of operators $T_{A_{s}}$ is continuous with respect to the gap topology. The eigenvalues of the family $T_{A_{s}}$ can be organized in smooth families

$$
\lambda_{s, n}=\frac{2 \pi}{L}\left(\omega_{s}+n\right)=\frac{2 \pi}{L}\left(\omega_{A_{0}}+s\left(\omega_{1}-\omega_{0}\right)+n\right), \omega_{s}:=\omega_{A_{s}}, \quad \forall s \in[0,1] .
$$


Assume for simplicity that $\omega_{0}, \omega_{1} \notin \mathbb{Z}$, i.e., the operators $T_{A_{0}}$ and $T_{A_{1}}$ are invertible. Denote by $S F\left(A_{1}, A_{0}\right)$ the spectral flow of the affine family $3 T_{A_{s}}$. Then

$$
\begin{gathered}
S F\left(A_{1}, A_{0}\right)=\#\left\{n \in \mathbb{Z} ; \quad-\omega_{1}<n<-\omega_{0}\right\}-\#\left\{n \in \mathbb{Z} ;-\omega_{0}<n<-\omega_{1}\right\} \\
=\#\left(\mathbb{Z} \cap\left(\omega_{0}, \omega_{1}\right)\right)-\#\left(\mathbb{Z} \cap\left(\omega_{1}, \omega_{0}\right)\right) .
\end{gathered}
$$

We conclude

$$
S F\left(A_{1}, A_{0}\right)=\left(\left\lfloor\omega_{1}\right\rfloor-\left\lfloor\omega_{0}\right\rfloor\right), \omega_{i}=\frac{A_{i}(L)}{2 \pi} .
$$

Using (1.10) we deduce

$$
S F\left(A_{1}, A_{0}\right)=\left\lfloor\omega_{A_{1}}\right\rfloor-\left\lfloor\omega_{A_{0}}\right\rfloor=\omega_{A_{1}}-\omega_{A_{0}}+\left(\xi_{A_{1}}-\xi_{A_{0}}\right) .
$$

Remark 1.6 (The rescaling trick). Note that the rescaling

$$
\left[0, L_{1}\right] \ni \tau \mapsto t=\frac{\tau}{c} \in\left[0, L_{0}\right], \quad c=\frac{L_{1}}{L_{0}}
$$

induces an isometry $\mathcal{J}_{L_{1}, L_{0}}: \boldsymbol{H}_{L_{0}}=L^{2}\left(\left[0, L_{0}\right] ; \mathbb{C}\right) \rightarrow \boldsymbol{H}_{L_{1}}=L^{2}\left(\left[0, L_{1}\right] ; \mathbb{C}\right)$,

$$
\boldsymbol{H}_{L_{0}} \ni f(t) \mapsto \mathcal{J}_{L_{1}, L_{0}} f(\tau):=c^{-1 / 2} f\left(\frac{\tau}{c}\right) \in \boldsymbol{H}_{L_{1}} .
$$

The unbounded operator $\frac{d}{d t}$ on $\boldsymbol{H}_{L_{0}}$ is the conjugate to the operator $c \frac{d}{d \tau}$ on $\boldsymbol{H}_{L_{1}}$.

If $\alpha(t)$ is a real bounded measurable function on $\left[0, L_{0}\right]$, then the bounded operator on $\boldsymbol{H}_{L_{0}}$ defined by pointwise multiplication by $\alpha(t)$ is conjugate to the bounded operator on $\boldsymbol{H}_{L_{1}}$ defined by the multiplication by $a(\tau)=\alpha(\tau / c)$. Hence the unbounded operator $D_{b}$ on $\boldsymbol{H}_{L_{0}}$ is conjugate to the unbounded operator $c D_{c^{-1} a}$ on $\boldsymbol{H}_{L_{1}}$,

$$
c D_{c^{-1} a}=\mathcal{J}_{L_{1}, L_{0}} D_{\alpha} \mathcal{J}_{L_{1}, L_{0}}^{-1} .
$$

Its resolvent is obtained by solving the periodic boundary value problem

$$
\boldsymbol{i} u+c\left(-\boldsymbol{i} \frac{d}{d \tau}+c^{-1} a(\tau)\right) u(\tau)=f(\tau), u(0)=u\left(L_{1}\right) .
$$

If we set

$$
A(\tau)=\int_{0}^{\tau} a(\sigma) d \sigma \text { and } \Phi_{A, c}(t)=c^{-1} \Phi_{A}(\tau)=c^{-1}(\boldsymbol{i} A(\tau)-\tau),
$$

then we see that $R_{\alpha}$ is conjugate to the integral operator $R_{A, c}$,

$$
R_{A, c} f(\tau)=\frac{c^{-1} \boldsymbol{i}^{-\Phi_{A, c}(\tau)}}{e^{\Phi_{A, c}\left(L_{1}\right)}-1} \int_{0}^{L_{1}} e^{\Phi_{A, c}(\sigma)} f(s) d s+c^{-1} \boldsymbol{i} \int_{0}^{t} e^{-\left(\Phi_{A, c}(\tau)-\Phi_{A}(\sigma)\right.} f(\sigma) d \sigma .
$$

Arguing exactly as in the proof of Proposition 1.3 we deduce that if $A_{n}$ converges very weakly to $A \in \mathcal{A}_{L_{1}}$ and the sequence of positive numbers $c_{n}$ converges to the positive number $c$, then $R_{A_{n}, c_{n}}$ converges in the operator norm to $R_{A, c}$.

For any $c>0$ and $A \in \mathcal{A}$ we define the operator

$$
T_{A, c}=R_{A, c}^{-1}-i, c>0 .
$$

Note that $T_{A, c}=c T_{c^{-1} A}$. Then for every $c>0$ the spectrum of $T_{A, c}$ is

$$
\operatorname{spec}\left(T_{A, c}\right)=c \operatorname{spec}\left(T_{c^{-1} A}\right) .
$$

\footnotetext{
${ }^{3}$ The quantity $S F\left(A_{1}, A_{0}\right)$ is independent of the weakly continuous path $A_{s}$ connecting $A_{0}$ to $A_{1}$ since the space $\mathcal{A}$ equipped with the weak topology is contractible. It is thus an invariant of the pair $\left(A_{1}, A_{0}\right)$.
} 
We want to give a more intuitive description of the operators $R_{A}$ and $T_{A}$ for a large class of $A$ 's. We begin by introducing a nice subclass $\mathcal{A}_{*}$ of $\mathcal{A}$. Let $H(t)$ denote the Heaviside function

$$
H(t)= \begin{cases}1, & t \geq 0 \\ 0, & t<0\end{cases}
$$

Definition 1.7. We say that $A \in \mathcal{A}$ is nice if there exists $a \in L^{\infty}(0, L)$, a finite subset $\mathcal{P} \subset(0, L)$, and a function $c: \mathcal{P} \rightarrow \mathbb{R}$ such that

$$
A(t)=A_{*}(t)+\sum_{p \in \Delta} c(p) H(t-p), \quad \forall t \in[0, L], \quad A_{*}(t):=\int_{0}^{t} a(s) d s .
$$

We denote by $\mathcal{A}_{*}$ the subcollection of nice functions.

Let us first point out that $\mathcal{A}_{*}$ is a vector subspace of $\mathcal{A}$. Next, observe that $A \in \mathcal{A}^{*}$ if and only if there exists a finite subset $\mathcal{P}_{A} \subset(0, L)$ such that the restriction of $A$ to $[0, L] \backslash \mathcal{P}$ is Lipschitz continuous. The function $A$ admits left and right limits at any point $t \in[0, L]$, and we define the jump function

$$
c: \mathcal{P}_{A} \rightarrow \mathbb{R}, \quad c(p)=\lim _{t \searrow p} A(t)-\lim _{t \nearrow p} A(t) .
$$

Then

$$
A_{*}(t)=A(t)-\sum_{p \in \mathcal{P}} c(p) H(t-p)
$$

is Lipschitz continuous, it is differentiable a.e. on $[0, L]$, and we define $a$ to be the derivative of $A_{*}$.

Let us next observe that if $A \in \mathcal{A}_{*}$, then the operator $T_{A}$ can be informally described as

$$
T_{A}=-i \frac{d}{d t}+a(t)+\sum_{p \in \mathcal{P}_{A}} c(p) \delta_{p}
$$

In other words, $T_{A}$ would like to be a Dirac-type operator whose coefficients are measures.

We will now give a precise description of $T_{A}$ as a closed unbounded selfadjoint operator defined by an elliptic boundary value problem. We need to introduce some more terminology.

For any $u$ defined on an interval $\left[a_{-}, a_{+}\right], a_{-}<a_{+}$, and any $x \in\left(a_{-}, a_{+}\right)$we set

$$
\gamma_{ \pm} u:=u\left(a_{ \pm}\right), u(x+0):=\lim _{t \searrow x} u(t), u(x-0):=\lim _{t \nearrow x} u(t) .
$$

We will say that $a_{ \pm}$is the outgoing/incoming boundary of the interval. For any partition of $[0, L], \mathcal{P}=\left\{0<t_{1}<\cdots<t_{n-1}<L\right\}$, we set

$$
t_{0}:=0, \quad t_{n}:=L, \quad I_{k}:=\left[t_{k-1}, t_{k}\right], \quad k=1, \ldots, n .
$$

We define the Hilbert space

$$
\boldsymbol{H}_{\mathcal{P}}:=\bigoplus_{k=1}^{n} L^{2}\left(I_{k}, \mathbb{C}\right)
$$

and the Hilbert space isomorphism

$$
\mathcal{J}_{\mathcal{P}}: \boldsymbol{H} \rightarrow \boldsymbol{H}_{\mathcal{P}}, \quad \boldsymbol{H} \ni f \mapsto\left(\left.f\right|_{I_{1}}, \ldots,\left.f\right|_{I_{n}}\right) \in \boldsymbol{H}_{\mathcal{P}}
$$


Let $A \in \mathcal{A}_{*}$ and $\mathcal{P}=\left\{0<t_{1}<\cdots<t_{n-1}<L\right\}$ be a partition that contains the set of discontinuities of $\mathcal{A}, \mathcal{P} \supset \mathcal{P}_{A}$. We set

$$
a=\frac{d A_{*}}{d t}, a_{k}=\left.a\right|_{I_{k}}, k=1, \ldots, n .
$$

For $j=1, \ldots, n-1$ we denote by $c_{j}=c_{j}(A)$ the jump of $A$ at $t_{j}$. Finally, we define the closed unbounded linear operator

$$
L_{\mathcal{A}, \mathcal{P}}: \operatorname{Dom}\left(L_{A, \mathcal{P}}\right) \subset \boldsymbol{H}_{\mathcal{P}} \rightarrow \boldsymbol{H}_{\mathcal{P}}
$$

where $\operatorname{Dom}\left(L_{A, \mathcal{P}}\right)$ consists of $n$-tuples $\left(u_{k}\right)_{1 \leq k \leq n} \in \boldsymbol{H}_{\mathcal{P}}$ such that

$$
\begin{gathered}
u_{k} \in L^{1,2}\left(I_{k}\right), \quad k=1, \ldots, n, \\
\gamma_{-} u_{j+1}=e^{-i c_{j}} \gamma_{+} u_{j}, \quad j=1, \ldots, n-1, \\
u_{n}(L)=u_{1}(0),
\end{gathered}
$$

and

$$
L_{A, \mathcal{P}}\left(u_{1}, \ldots, u_{n}\right)=\left(-i \frac{d u_{1}}{d t}+a_{1} u_{1}, \ldots,-i \frac{d u_{n}}{d t}+a_{n} u_{n}\right) .
$$

A standard argument shows that $L_{A, \mathcal{P}}$ is closed, densely defined and selfadjoint. In particular, the operator $\left(L_{A, \mathcal{P}}+\boldsymbol{i}\right)$ is invertible, with bounded inverse.

Theorem 1.8. For any $A \in \mathcal{A}_{*}$ and any partition $\mathcal{P}=\left\{0<t_{1}<\cdots<t_{n-1}<L\right\}$ that contains the set of discontinuities of $\mathcal{A}$ we have the equality

$$
L_{A, \mathcal{P}}=\mathcal{J}_{\mathcal{P}} T_{A} \mathcal{J}_{\mathcal{P}}^{-1} .
$$

Proof. For simplicity we write $L_{A}$ instead of $L_{A, \mathcal{P}}$. We will prove the equivalent statement

$$
\left(\boldsymbol{i}+L_{A}\right)^{-1}=\mathcal{J}_{\mathcal{P}}\left(T_{A}+\boldsymbol{i}\right)^{-1} \mathcal{J}_{\mathcal{P}}^{-1}=\mathcal{J}_{\mathcal{P}} R_{A} \mathcal{J}_{\mathcal{P}}^{-1} .
$$

In other words, we have to prove that for any $u, f \in \boldsymbol{H}$, if $u=R_{A} f$, then

$$
u \in \operatorname{Dom}\left(L_{A}\right) \text { and }\left(L_{A}+\boldsymbol{i}\right) \mathcal{J}_{\mathcal{P}} u=\mathcal{J}_{\mathcal{P}} f .
$$

More precisely, we have to show that the collection $\mathcal{J}_{A} u=\left(u_{k}\right)_{1 \leq k \leq n}$ satisfies (1.14a) - (1.14c) and (1.15). Using (1.2) we deduce

$$
u(t)=\frac{\boldsymbol{i} e^{-\Phi_{A}(t)}}{e^{\Phi_{A}(L)}-1} \int_{0}^{L} e^{\Phi_{A}(s)} f(s) d s+\boldsymbol{i} e^{-\Phi_{A}(t)} \int_{0}^{t} e^{\Phi_{A}(s)} f(s) d s .
$$

This implies the condition (1.14a). The condition (1.15) follows by direct computation using (1.16).

Next, we observe that

$$
\begin{gathered}
\gamma_{+} u_{j}=\frac{\boldsymbol{i} e^{-\Phi_{A}\left(t_{j}-0\right)}}{e^{\Phi_{A}(L)}-1} \int_{0}^{L} e^{\Phi_{A}(s)} f(s) d s+\boldsymbol{i} e^{-\Phi_{A}\left(t_{j}-0\right)} \int_{0}^{t_{j}} e^{\Phi_{A}(s)} f(s) d s, \\
\gamma_{-} u_{j+1}=\frac{\boldsymbol{i} e^{-\Phi_{A}\left(t_{j}+0\right)}}{e^{\Phi_{A}(L)}-1} \int_{0}^{L} e^{\Phi_{A}(s)} f(s) d s+\boldsymbol{i} e^{-\Phi_{A}\left(t_{j}+0\right)} \int_{0}^{t_{j}} e^{\Phi_{A}(s)} f(s) d s,
\end{gathered}
$$

from which we conclude that

$$
\gamma_{-} u_{j+1}=e^{-\left(\Phi_{A}\left(t_{j}+0\right)-\Phi_{A}\left(t_{j}-0\right)\right)} \gamma_{+} u_{j}, \quad \forall j=1, \ldots, n-1 .
$$

This proves (1.14b). The equality (1.14c) follows directly from (1.5). 
Remark 1.9 (Transmission operators). We would like to place the above operator $L_{A}$ in a broader perspective that we will use extensively in Section 4 . Consider a compact, oriented 1-dimensional manifold with boundary $\boldsymbol{I}$. In other words, $\boldsymbol{I}$ is a disjoint union of finitely many compact intervals

$$
\boldsymbol{I}=\bigsqcup_{k=1}^{n} I_{k}
$$

If $I_{k}:=\left[a_{k}, b_{k}\right], a_{k}<b_{k}$, then we set

$$
\partial_{+} I_{k}:=\left\{b_{k}\right\}, \partial_{-} I_{k}:=\left\{a_{k}\right\}, \partial_{+} \boldsymbol{I}:=\left\{b_{1}, \ldots, b_{n}\right\}, \partial_{-} \boldsymbol{I}:=\left\{a_{1}, \ldots, a_{n}\right\} .
$$

In particular, we have a direct sum decomposition of (finite-dimensional) Hilbert spaces

$$
\boldsymbol{E}:=L^{2}(\partial \boldsymbol{I}, \mathbb{C})=L^{2}\left(\partial_{+} \boldsymbol{I}\right) \oplus L^{2}\left(\partial_{-} \boldsymbol{I}\right)=: \boldsymbol{E}_{+} \oplus \boldsymbol{E}_{-} .
$$

On the space $C^{\infty}(\boldsymbol{I}, \mathbb{C})$ of smooth complex-valued functions on $\boldsymbol{I}$ we have a canonical, symmetric Dirac $\mathcal{D}$ operator described on each $I_{k}$ by $-\boldsymbol{i} \frac{d}{d t}$. We have a natural operator

$$
J: L^{2}(\partial \boldsymbol{I}, \mathbb{C}) \rightarrow L^{2}(\partial \boldsymbol{I}, \mathbb{C}),\left.\quad J\right|_{\boldsymbol{E}_{ \pm}}=\mp \boldsymbol{i} \mathbb{1}_{\boldsymbol{E}_{ \pm}} .
$$

It thus defines a Hermitian symplectic structure in the sense of [1, 5, 14. A (Hermitian) Lagrangian subspace of $\boldsymbol{E}$ is then a complex subspace $L$ such that $L^{\perp}=J L$. We denote by $\boldsymbol{L a g}(\boldsymbol{E}, J)$ the Grassmannin of Hermitian Lagrangian spaces. We denote by $\operatorname{Iso}\left(\boldsymbol{E}_{+}, \boldsymbol{E}_{-}\right)$the space of linear isometries $\boldsymbol{E}_{+} \rightarrow \boldsymbol{E}_{-}$. As explained in [1] there exists a natural bijection 4

$$
\operatorname{Iso}\left(\boldsymbol{E}_{+}, \boldsymbol{E}_{-}\right) \rightarrow \boldsymbol{L a g}(\boldsymbol{E}), \quad \mathrm{Iso}\left(\boldsymbol{E}_{+}, \boldsymbol{E}_{-}\right) \ni T \longmapsto \Gamma_{T},
$$

where $\Gamma_{T}$ is the graph of $T$ viewed as a subspace of $\boldsymbol{E}$. Our spaces $\boldsymbol{E}_{ \pm}$are equipped with natural bases and through these bases we can identify $\operatorname{Iso}\left(\boldsymbol{E}_{+}, \boldsymbol{E}_{-}\right)$with the unitary group $U(n)$. We denote by $\Delta$ the Lagrangian subspace corresponding to the identity operator.

Any subspace $V \subset \boldsymbol{E}$ defines a Fredholm operator

$$
\mathcal{D}_{V}: \operatorname{Dom}\left(\mathcal{D}_{V}\right) \subset L^{2}(\boldsymbol{I}, \mathbb{C}) \rightarrow L^{2}(\boldsymbol{I}, \mathbb{C}),
$$

where

$$
\operatorname{Dom}\left(\mathcal{D}_{V}\right)=\left\{u \in L^{1,2}(\boldsymbol{I}, \mathbb{C}) ;\left.u\right|_{\partial I} \in V\right\}, \quad \mathcal{D}_{V} u=\mathcal{D} u .
$$

A simple argument shows that $\mathcal{D}_{V}$ is selfadjoint if and only if $V \in \boldsymbol{L a g}(\boldsymbol{E})$. As we explained above, in this case $V$ can be identified with the graph of an isometry $T: \boldsymbol{E}_{+} \rightarrow \boldsymbol{E}_{-}$. We say that $T$ is the transmission operator associated to the selfadjoint boundary value problem.

For example, if in Theorem 1.8 we let $A(t)=\sum_{j=1}^{n-1} c_{j} H\left(t-t_{j}\right)$, then we see that the operator $L_{A}$ can be identified with the operator $\mathcal{D}_{\Gamma_{T}}$, where the transmission

${ }^{4}$ There are various conventions in the definition of this bijection. We follow the conventions in 
operator $T \in \operatorname{Iso}\left(\boldsymbol{E}_{+}, \boldsymbol{E}_{-}\right)$is given by the unitary $n \times n$ matrix

$$
T=\left[\begin{array}{llcccc}
0 & 0 & 0 & \cdots & 0 & 1 \\
e^{-i c_{1}} & 0 & 0 & \cdots & 0 & 0 \\
0 & e^{-i c_{2}} & 0 & \cdots & 0 & 0 \\
\vdots & \vdots & \vdots & \vdots & \vdots & \vdots \\
\cdot & \cdot & \cdot & \cdot & \cdot & \cdot \\
0 & 0 & 0 & \cdots & e^{-i c_{n-1}} & 0
\end{array}\right] .
$$

\section{The Atiyah-Patodi-Singer theorem}

Here we review the Atiyah-Patodi-Singer index theorem for Dirac operators on a manifold with boundary, when the metric is not assumed to be cylindrical near the boundary. Our presentation closely follows [8, 9, but we present a few more details since the various orientation conventions and the terminology in [8, 9] are different from those in 3,13 , that we use throughout this paper.

Suppose $(\widehat{M}, \hat{g})$ is compact, oriented Riemann, and let $M \subset \widehat{M}$ be a hypersurface in $\widehat{M}$ co-oriented by a unit normal vector field $\boldsymbol{\nu}$ along $M$. Let $n:=\operatorname{dim} M$ so that $\operatorname{dim} \widehat{M}=n+1$. We denote by $g$ the induced metric on $M$. We first want to define a canonical restriction to $M$ of a Dirac operator on $\hat{M}$.

Let $\exp ^{\hat{g}}: T \widehat{M} \rightarrow \widehat{M}$ denote the exponential map determined by the metric $\hat{H}$. For sufficiently small $\varepsilon>0$ the map

$$
(-\varepsilon, \varepsilon) \times M \ni(t, p) \mapsto \exp _{p}^{\hat{g}}(t \boldsymbol{\nu}(p)) \in \widehat{M}
$$

is a diffeomorphism onto a small open tubular neighborhood $\mathcal{O}_{\varepsilon}$ of $M$. The metric $g$ determines a cylindrical metric $d t^{2}+g$ on $(-\varepsilon, \varepsilon) \times M$. Via the above diffeomorphism we get a metric $\hat{g}_{0}$ on $\mathcal{O}_{\varepsilon}$. We say that $\hat{g}_{0}$ is the cylindrical approximation of $\hat{g}$ near $M$.

We denote by $\widehat{\nabla}$ the Levi-Civita connection of the metric $\hat{g}$ and by $\widehat{\nabla}^{0}$ the LeviCivita connection of the metric $\hat{g}_{0}$. We set

$$
\boldsymbol{\Xi}:=\widehat{\nabla}-\widehat{\nabla}^{0} \in \Omega^{1}\left(\mathcal{O}_{\varepsilon}, \operatorname{End}(T \widehat{M})\right) .
$$

To get a more explicit description of $\boldsymbol{\Xi}$ we fix a local oriented, $g$-orthonormal frame $\left(\boldsymbol{e}_{1}, \ldots, \boldsymbol{e}_{n}\right)$ on $M$. Together with the unit normal vector field $\boldsymbol{\nu}$ we obtain a local oriented orthonormal frame $\left(\boldsymbol{\nu}, \boldsymbol{e}_{1}, \ldots, \boldsymbol{e}_{n}\right)$ of $\left.T \widehat{M}\right|_{M}$. We extend it by parallel transport along the geodesics orthogonal to $M$ to a local, oriented orthonormal frame $\left(\hat{\boldsymbol{\nu}}, \hat{\boldsymbol{e}}_{1}, \ldots, \hat{\boldsymbol{e}}_{n}\right)$ of $T \widehat{M}$.

Denote by $\widehat{\boldsymbol{\omega}}$ the connection form associated to $\widehat{\nabla}$ by this frame, and by $\widehat{\boldsymbol{\theta}}$ the connection form associated to $\widehat{\nabla}^{0}$ by this frame. We can represent both $\widehat{\boldsymbol{\omega}}$ and $\widehat{\boldsymbol{\theta}}$ as skew-symmetric $(n+1) \times(n+1)$ matrices

$$
\widehat{\boldsymbol{\omega}}=\left(\widehat{\boldsymbol{\omega}}_{j}^{i}\right)_{0 \leq i, j \leq n}, \quad \widehat{\boldsymbol{\theta}}=\left(\widehat{\boldsymbol{\theta}}_{j}^{i}\right)_{0 \leq i, j \leq n},
$$

where the entries are 1-forms. Then $\boldsymbol{\Xi}=\widehat{\boldsymbol{\omega}}-\widehat{\boldsymbol{\theta}}$.

We set $\hat{\boldsymbol{e}}_{0}:=\hat{\boldsymbol{\nu}}$, and we denote by $\left(\hat{\boldsymbol{e}}^{k}\right)_{0 \leq k \leq n}$ the dual orthonormal frame of $T^{*} \widehat{M}$. Then we have

$$
\hat{\boldsymbol{\omega}}_{j}^{i}=\hat{\boldsymbol{\omega}}_{k j}^{i} \hat{\boldsymbol{e}}^{k}, \quad \hat{\boldsymbol{\theta}}_{j}^{i}=\hat{\boldsymbol{\theta}}_{k j}^{i} \hat{\boldsymbol{e}}^{k}, \quad \hat{\nabla}_{k} \hat{\boldsymbol{e}}_{j}=\hat{\boldsymbol{\omega}}_{k j}^{i} \hat{\boldsymbol{e}}_{i}, \quad \widehat{\nabla}_{k}^{0} \hat{\boldsymbol{e}}_{j}=\hat{\boldsymbol{\theta}}_{k j}^{i} \hat{\boldsymbol{e}}_{i}, \quad \forall 0 \leq j, k \leq n,
$$

where we have used Einstein's summation convention. 
Observe that $\widehat{\nabla}^{0} \hat{\boldsymbol{e}}_{0}=0$ so that $\hat{\boldsymbol{\theta}}_{0}^{i}=\hat{\boldsymbol{\theta}}_{i}^{0}=0$. Also,

$$
\hat{\boldsymbol{\omega}}_{j k}^{i}=\hat{\boldsymbol{\theta}}_{j k}^{i}, \quad \forall 1 \leq i, j, k \leq n .
$$

If we write

$$
\boldsymbol{\Xi}=\left(\boldsymbol{\Xi}_{j}^{i}\right)_{0 \leq i, j \leq n}, \quad \boldsymbol{\Xi}_{j}^{i}=\boldsymbol{\Xi}_{j k}^{i} \hat{\boldsymbol{e}}^{k},
$$

and we let $o(1)$ denote any quantity that vanishes along $M$, then

$$
\begin{gathered}
\boldsymbol{\Xi}_{j}^{i}=-\boldsymbol{\Xi}_{i}^{j}, \quad \forall 0 \leq i, j \leq n, \\
\boldsymbol{\Xi}_{k j}^{i}=o(1), \quad \forall 1 \leq i, j \leq n, \quad 0 \leq k \leq n .
\end{gathered}
$$

We set

$$
\boldsymbol{\Xi}_{k i j}:=\boldsymbol{\Xi}_{k j}^{i}=\hat{g}\left(\hat{\nabla}_{k} \hat{\boldsymbol{e}}_{j}, \hat{\boldsymbol{e}}_{i}\right), \quad \hat{\boldsymbol{\omega}}_{i j}:=\hat{\boldsymbol{\omega}}_{j}^{i}, \hat{\boldsymbol{\theta}}_{i j}:=\hat{\boldsymbol{\theta}}_{j}^{i}, \quad \boldsymbol{\omega}_{k i j}:=\boldsymbol{\omega}_{k j}^{i}, \quad \boldsymbol{\theta}_{k i j}:=\boldsymbol{\theta}_{k j}^{i} .
$$

We denote by $Q$ the second fundamental form 5 of the embedding $M \hookrightarrow \widehat{M}$,

$$
Q\left(e_{i}, e_{j}\right)=g\left(\widehat{\nabla}_{\boldsymbol{e}_{i}} \boldsymbol{\nu}, \boldsymbol{e}_{j}\right) .
$$

Along the boundary we have the equalities

$$
\begin{gathered}
\Xi_{k j 0}=\boldsymbol{\Xi}_{j k 0}=-\boldsymbol{\Xi}_{k 0 j}=Q\left(\boldsymbol{e}_{j}, \boldsymbol{e}_{k}\right) \quad \forall 1 \leq i, j \leq n, \\
\Xi_{i j 0}=0, \quad \forall 0 \leq i, j \leq n .
\end{gathered}
$$

To understand the nature of the restriction to a hypersurface of a Dirac operator we begin with a special case. Namely, we assume that $\widehat{M}$ is equipped with a spin structure. We denote by $\widehat{\mathbb{S}}$ the associated complex spinor bundle so that $\widehat{\mathbb{S}}$ is $\mathbb{Z} / 2$ graded if $\operatorname{dim} \widehat{M}$ is even, and ungraded otherwise. We have a Clifford multiplication

$$
\hat{\boldsymbol{c}}: T^{*} M \rightarrow \operatorname{End}(\hat{\mathbb{S}}) .
$$

The metrics $\hat{g}$ and $\hat{g}_{0}$ define connections $\widehat{\nabla}^{\text {spin }}$ and $\widehat{\nabla}$ spin,0 on $\left.\widehat{\mathbb{S}}\right|_{\mathcal{O}_{\varepsilon}}$. Using the local frame $\left(\hat{\boldsymbol{e}}_{i}\right)_{0 \leq i, j \leq n}$ we can write

$$
\widehat{\nabla}_{k}^{s p i n}=\partial_{k}-\frac{1}{4} \hat{\boldsymbol{\omega}}_{k i j} \hat{\boldsymbol{c}}\left(\hat{\boldsymbol{e}}^{i}\right) \hat{\boldsymbol{c}}\left(\hat{\boldsymbol{e}}^{j}\right), \quad \widehat{\nabla}_{k}^{s p i n, 0}=\partial_{k}-\frac{1}{4} \hat{\boldsymbol{\theta}}_{k i j} \hat{\boldsymbol{c}}\left(\hat{\boldsymbol{e}}^{i}\right) \hat{\boldsymbol{c}}\left(\hat{\boldsymbol{e}}^{j}\right),
$$

where we again use Einstein's summation convention.

Using the connections $\widehat{\nabla}^{\text {spin }}$ and $\widehat{\nabla}^{\text {spin, }, 0}$ we obtain two Dirac operators $\hat{D}$ and respectively $\hat{D}_{0}$ on $\left.\widehat{\mathbb{S}}\right|_{\mathcal{O}_{\varepsilon}}$,

$$
\hat{D}=\sum_{i=0}^{n} \hat{\boldsymbol{c}}\left(\hat{e}^{i}\right) \widehat{\nabla}_{i}^{s p i n}, \quad \hat{D}_{0}=\sum_{i=0}^{n} \hat{\boldsymbol{c}}\left(\hat{e}^{i}\right) \widehat{\nabla}_{i}^{s p i n, 0} .
$$

Identifying $\mathcal{O}_{\varepsilon}$ with $(-\varepsilon, \varepsilon) \times M$ we obtain a projection $\pi: \mathcal{O}_{\varepsilon} \rightarrow M$. We set $\mathbb{S}:=\left.\hat{\mathbb{S}}\right|_{M}$. The parallel transport given by $\hat{\nabla}^{\text {spin }}$ yields a bundle isomorphism $\left.\hat{\mathbb{S}}\right|_{\mathcal{O}_{\varepsilon}} \cong \pi^{*} \mathbb{S}$. Using these identifications we can rewrite the operators $\hat{D}$ and $\hat{D}_{0}$ as

$$
\begin{gathered}
\hat{D}=\hat{\boldsymbol{c}}\left(\hat{\boldsymbol{e}}_{0}\right)\left(\hat{\nabla}_{0}^{\text {spin }}-D(t)\right): C^{\infty}\left(\pi^{*} \mathbb{S}\right) \rightarrow C^{\infty}\left(\pi^{*} \mathbb{S}\right), \\
\hat{D}_{0}=\hat{\boldsymbol{c}}\left(\hat{\boldsymbol{e}}^{0}\right)\left(\partial_{0}-D_{0}(t)\right): C^{\infty}\left(\pi^{*} \mathbb{S}\right) \rightarrow C^{\infty}\left(\pi^{*} \mathbb{S}\right) .
\end{gathered}
$$

\footnotetext{
${ }^{5}$ Our definition of the second fundamental form differs by a sign and a factor from the usual definition. With our definition the round sphere $S^{n} \subset \mathbb{R}^{n+1}$ co-oriented by the outer normal has positive mean curvature $n$.
} 
The operators $D(t)$ and $D_{0}(t)$ are first order differential operators

$$
C^{\infty}\left(\left.\widehat{\mathbb{S}}\right|_{\{t\} \times M}\right) \rightarrow C^{\infty}\left(\left.\widehat{\mathbb{S}}\right|_{\{t\} \times M}\right),
$$

and thus can be viewed as $t$-dependent operators on $\mathbb{S}$.

The operator $D_{0}(t)$ is in fact independent of $t$, and thus we can identify it with a Dirac operator on $C^{\infty}(\mathbb{S}) \rightarrow C^{\infty}(M)$. It is called the canonical restriction of $\hat{D}$ to $M$, and we will denote it by $\mathcal{R}_{M}(\widehat{D})$. This operator is intrinsic to $M$. More precisely, when $\operatorname{dim} \widehat{M}$ is even, then $\mathbb{S}$ is the direct sum of two copies of the spinor bundle on $M$ and the operator $\mathcal{R}_{M}(\widehat{D})$ is the direct sum of two copies of the spin-Dirac operator determined by the Riemann metric on $M$. When $\operatorname{dim} \widehat{M}$ is odd, then $\mathbb{S}$ is the spinor bundle on $M$ and $\mathcal{R}_{M}(\widehat{D})$ is the spin-Dirac operator determined by the metric on the boundary and the induced spin structure. We would like to express $\mathcal{R}_{M}(\hat{D})$ in terms of $\left.D(t)\right|_{t=0}$.

Lemma 2.1. Let $h_{M}: M \rightarrow \mathbb{R}$ be the mean curvature of $M \hookrightarrow \widehat{M}$, i.e., the scalar $h_{M}:=\operatorname{tr} Q$. Then,

$$
\left.D(t)\right|_{t=0}=\mathcal{R}_{M}(\widehat{D})-\frac{1}{2} h_{M}
$$

Proof. Let $\boldsymbol{\nu}_{*}:=\hat{\boldsymbol{e}}^{0} \in C^{\infty}\left(\left.T^{*} \widehat{M}\right|_{\partial \widehat{M}}\right)$, set $J:=\hat{\boldsymbol{c}}\left(\boldsymbol{\nu}_{*}\right)$ and define

$$
c: T^{*} M \rightarrow \operatorname{End}(\mathbb{S})
$$

by setting

$$
\boldsymbol{c}(\alpha):=\hat{\boldsymbol{c}}\left(\boldsymbol{\nu}_{*}(p)\right) \hat{\boldsymbol{c}}(\alpha)=J \hat{\boldsymbol{c}}(\alpha), \quad \forall p \in M,\left.\quad \alpha \in T^{*} M \subset\left(T^{*} \widehat{M}\right)\right|_{M .}
$$

Observe first that

$$
\mathcal{R}_{M}(\hat{D})=D_{0}(t)=\partial_{0}+J \hat{D}_{0} .
$$

Next we observe that

$$
\widehat{\nabla}^{\text {spin }}-\widehat{\nabla}^{\text {spin }, 0}=-\frac{1}{4} \sum_{i, j} \boldsymbol{\Xi}_{i j} \hat{\boldsymbol{c}}\left(\hat{\boldsymbol{e}}^{i}\right) \hat{\boldsymbol{c}}\left(\hat{\boldsymbol{e}}^{j}\right)
$$

so that

$$
\begin{aligned}
\widehat{\nabla}_{0}^{\text {spin }}-\widehat{\nabla}_{0}^{\text {spin }, 0} & =\widehat{\nabla}_{0}^{\text {spin }}-\partial_{0}=-\frac{1}{4} \Xi_{0 i j} J \hat{\boldsymbol{c}}\left(\hat{\boldsymbol{e}}^{i}\right) \hat{\boldsymbol{c}}\left(\hat{\boldsymbol{e}}^{j}\right)=o(1), \\
\hat{D}-\hat{D}_{0} & =-\frac{1}{4} \sum_{i, j, k} \boldsymbol{\Xi}_{k i j} \hat{\boldsymbol{c}}\left(\hat{\boldsymbol{e}}^{k}\right) \hat{\boldsymbol{c}}\left(\hat{\boldsymbol{e}}^{i}\right) \hat{\boldsymbol{c}}\left(\hat{\boldsymbol{e}}^{j}\right)=: \mathcal{L}
\end{aligned}
$$

We denote by $\mathcal{L}(t)$ the restriction of $\mathcal{L}$ to the slice $\{t\} \times M$ so that $\mathcal{L}(t)$ is an endomorphism of $\left.\widehat{\mathbb{S}}\right|_{\{t\} \times M}$. Hence

$$
\hat{D}=J \partial_{0}-J D(t), \quad D(t)=J \hat{D}+\partial_{0}=J \hat{D}_{0}+\partial_{0}+J \mathcal{L}=D_{0}(t)+J \mathcal{L},
$$

so that

$$
D(0)=\mathcal{R}_{M}(\hat{D})+\left.J \mathcal{L}(t)\right|_{t=0} .
$$

Thus, we need to compute the endomorphism $\left.J \mathcal{L}(t)\right|_{t=0}$. We have

$$
J \mathcal{L}=-\frac{1}{4} \sum_{i, j, k} J \boldsymbol{\Xi}_{k i j} \hat{\boldsymbol{c}}\left(\hat{\boldsymbol{e}}^{k}\right) \hat{\boldsymbol{c}}\left(\hat{\boldsymbol{e}}^{i}\right) \hat{\boldsymbol{c}}\left(\hat{\boldsymbol{e}}^{j}\right)
$$


There are many cancellations in the above sum. Using (2.2) we deduce that the terms corresponding to $k=0$ vanish. Using (2.1) we deduce that the terms corresponding to $i, j>0$ or $i=j$ also vanish along the boundary. Thus

$$
\begin{aligned}
J \mathcal{L} & =-\frac{1}{4} \sum_{i \neq j, k \neq 0} \boldsymbol{\Xi}_{k i j} J \hat{\boldsymbol{c}}\left(\hat{\boldsymbol{e}}^{k}\right) \hat{\boldsymbol{c}}\left(e^{i}\right) \hat{\boldsymbol{c}}\left(e^{j}\right)+o(1) \\
& =-\frac{1}{2} \sum_{i>j, k>0} \boldsymbol{\Xi}_{k i j} J \hat{\boldsymbol{c}}\left(\hat{\boldsymbol{e}}^{k}\right) \hat{\boldsymbol{c}}\left(e^{i}\right) \hat{\boldsymbol{c}}\left(e^{j}\right)+o(1) \\
& =-\frac{1}{2} \sum_{i>0, k>0} \boldsymbol{\Xi}_{k i 0} J \hat{\boldsymbol{c}}\left(\hat{\boldsymbol{e}}^{k}\right) \hat{\boldsymbol{c}}\left(\hat{\boldsymbol{e}}^{i}\right) \hat{\boldsymbol{c}}\left(\hat{\boldsymbol{e}}^{0}\right)+o(1) .
\end{aligned}
$$

Using the equalities $J=\hat{\boldsymbol{c}}\left(\hat{\boldsymbol{e}}^{0}\right), J \hat{\boldsymbol{c}}\left(\boldsymbol{e}^{\ell}\right)=-\hat{\boldsymbol{c}}\left(\hat{\boldsymbol{e}}^{k}\right) J$ for $\ell>0$ we deduce

$$
J \mathcal{L}=\frac{1}{2} \sum_{i, k>0} \boldsymbol{\Xi}_{k i 0} \hat{\boldsymbol{c}}\left(\boldsymbol{e}^{k}\right) \hat{\boldsymbol{c}}\left(\hat{\boldsymbol{e}}^{j}\right)=-\frac{1}{2} \sum_{j>0} \boldsymbol{\Xi}_{i i 0}+o(1)=-\frac{1}{2} \operatorname{tr} Q .
$$

This proves (2.4).

Remark 2.2. An equality similar to (2.4) was proved in [12, Lemma 4.5.1], although in 12 the authors use different conventions for the induced Clifford multiplication on the boundary that lead to some sign differences. That is why we chose to go through all of the above computational details.

If now $\widehat{E} \rightarrow \widehat{M}$ is a Hermitian vector bundle over $\widehat{M}$ and $\widehat{\nabla}^{E}$ is a Hermitian connection on $\widehat{E}$, then we obtain in standard fashion a twisted Dirac operator $\hat{D}_{E}: C^{\infty}(\widehat{\mathbb{S}} \otimes \widehat{E}) \rightarrow C^{\infty}(\widehat{\mathbb{S}} \otimes \widehat{E})$. Using the parallel transport given by $\widehat{\nabla}^{E}$ we obtain an isomorphism $\left.\widehat{E}\right|_{\mathcal{O}_{\varepsilon}} \cong \pi^{*} E$, where $E:=\left.\widehat{E}\right|_{M}$. Along $\mathcal{O}_{\varepsilon}$ the operator $\widehat{D}_{E}$ has the form

$$
\widehat{D}_{E}=J\left(\partial_{t}-D_{E}(t)\right)
$$

If on $\mathcal{O}_{\varepsilon}$ we replace the metric $\hat{g}$ with its cylindrical approximation $\hat{g}_{0}$, then we obtain a new Dirac operator

$$
\widehat{D}_{E, 0}: C^{\infty}\left(\pi^{*}(\mathbb{S} \otimes E)\right) \rightarrow C^{\infty}\left(\pi^{*}(\mathbb{S} \otimes E)\right),
$$

which near the boundary has the form $J\left(\partial_{t}-D_{E_{0}}\right)$, where

$$
D_{E, 0}: C^{\infty}(\mathbb{S} \otimes E) \rightarrow C^{\infty}(\mathbb{S} \otimes E) .
$$

We set $\mathcal{R}_{M}\left(\widehat{D}_{E}\right):=D_{E, 0}$ and as before we obtain the identity

$$
\left.D_{E}(t)\right|_{t=0}=\mathcal{R}_{M}\left(\widehat{D}_{E}\right)-\frac{1}{2} h_{M}
$$

This is a purely local result so that a similar formula holds for the geometric Dirac operators determined by a $\operatorname{spin}^{c}$ structure.

We want to apply the above discussion to a very special case. Consider a compact oriented surface $\Sigma$ with, possibly disconnected, boundary $\partial \Sigma$. We think of $\partial \Sigma$ as a hypersurface in $\Sigma$ co-oriented by the outer normal.

Fix a Riemann metric $\hat{g}$ on $\Sigma$, smooth up to the boundary. Denote by $s$ the arclength coordinate on a component $\partial_{0} \Sigma$ of the boundary. As before we can identify an open neighborhood $\mathcal{O}$ of this component of the boundary with a cylinder $(-\varepsilon, 0] \times S^{1}$. In this neighborhood the metric $\hat{g}$ has the form

$$
\hat{g}=d t^{2}+w^{2} d s^{2},
$$


where $w=w(t, s):(-\varepsilon, 0] \times S^{1} \rightarrow(0, \infty)$ is a smooth positive function in the variables $t, s$ such that $w(0, s)=1, \forall s$.

The metric and the orientation on $\Sigma$ define an integrable almost complex structure $J: T \Sigma \rightarrow T \Sigma$. More precisely, $J$ is given by the counterclockwise rotation by $\pi / 2$. We denote by $K_{\Sigma}$ the canonical complex line bundle determined by $J$. We get a Dolbeault operator

$$
\left(\bar{\partial}+\bar{\partial}^{*}\right): C^{\infty}\left(\underline{\mathbb{C}}_{\Sigma} \oplus K_{\Sigma}^{-1}\right) \rightarrow C^{\infty}\left(\underline{\mathbb{C}}_{\Sigma} \oplus K_{\Sigma}^{-1}\right) .
$$

This can be identified with the Dirac operator defined by the metric $\hat{g}$ and the $\operatorname{spin}^{c}$ structure determined by the almost complex structure $J$. The associated line bundle is $K_{\Sigma}^{-1}$, and the connection on $K_{\Sigma}^{-1}$ is the connection induced by the Levi-Civita connection of the metric $\hat{g}$.

Let us explain this identification on the cylindrical neighborhood $\mathcal{O}$. We set

$$
\boldsymbol{e}^{0}=d t, \quad \boldsymbol{e}^{1}=w d s .
$$

Then $\left\{\boldsymbol{e}^{0}, \boldsymbol{e}^{1}\right\}$ is an oriented, orthonormal frame of $\left.T^{*} \Sigma\right|_{\mathcal{O}}$. We denote by $\left\{\boldsymbol{e}_{0}, \boldsymbol{e}_{1}\right\}$ its dual frame of $T \Sigma$. We let $c: T^{*} \Sigma \rightarrow \operatorname{End}\left(\underline{\mathbb{C}}_{\Sigma} \oplus K_{\Sigma}\right)$ be the Clifford multiplication normalized by the condition that the operator $d V:=\boldsymbol{c}\left(\boldsymbol{e}^{0}\right) \boldsymbol{c}\left(\boldsymbol{e}^{1}\right)$ on $\mathbb{C}_{\Sigma} \oplus K_{\Sigma}^{-1}$ has the block decomposition [3, §3.2],

$$
\boldsymbol{c}\left(\boldsymbol{e}^{0}\right) \boldsymbol{c}\left(\boldsymbol{e}^{1}\right)=\left[\begin{array}{cc}
-\boldsymbol{i} & 0 \\
0 & \boldsymbol{i}
\end{array}\right]
$$

The Levi-Civita connection of the metric $\hat{g}$ induces a natural connection on $K_{\Sigma}^{-1}$, and if we use the trivial connection on $\underline{\mathbb{C}}_{\Sigma}$ we get a connection $\widetilde{\nabla}$ on $\underline{\mathbb{C}}_{\Sigma} \oplus K_{\Sigma}^{-1}$. The associated Dirac operator is $D_{\Sigma}=c \circ \widetilde{\nabla}$, and we have the equality $D_{\Sigma}=\sqrt{2}\left(\bar{\partial}+\bar{\partial}^{*}\right)$. The even part of this operator is

$$
D_{\Sigma}^{+}=\sqrt{2} \bar{\partial}: C^{\infty}\left(\underline{\mathbb{C}}_{\Sigma}\right) \rightarrow C^{\infty}\left(K_{\Sigma}^{-1}\right)
$$

We want to compute its canonical restriction to the boundary. If we denote by $\partial$ the trivial connection on $\underline{\mathbb{C}}_{\Sigma}$, then

$$
D_{\Sigma}^{+}:=\boldsymbol{c}\left(\boldsymbol{e}^{0}\right) \partial_{\boldsymbol{e}_{0}}+\boldsymbol{c}\left(\boldsymbol{e}^{1}\right) \partial_{\boldsymbol{e}_{1}}=\boldsymbol{c}\left(\boldsymbol{e}_{0}\right)\left(\partial_{t}-\boldsymbol{c}\left(\boldsymbol{e}^{0}\right) \boldsymbol{c}\left(\boldsymbol{e}^{1}\right) \partial_{\boldsymbol{e}_{1}}\right)
$$

so that

$$
D_{\Sigma}^{+}(t)=\boldsymbol{c}\left(\boldsymbol{e}^{0}\right) D_{\Sigma}^{+}+\partial_{t}=\boldsymbol{c}\left(\boldsymbol{e}^{0}\right) \boldsymbol{c}\left(\boldsymbol{e}^{1}\right) \partial_{\boldsymbol{e}_{1}} \stackrel{2.6}{=}-\boldsymbol{i} \partial_{\boldsymbol{e}_{1}} .
$$

Above, the operator $D_{\Sigma}^{+}(t)$ is, canonically, a differential operator

$$
D_{\Sigma}^{+}(t): C^{\infty}\left(\underline{\mathbb{C}}_{\partial \Sigma}\right) \rightarrow C^{\infty}\left(\underline{\mathbb{C}}_{\partial \Sigma}\right),
$$

where $\underline{\mathbb{C}}_{\partial \Sigma}$ denotes the trivial complex line bundle over $\partial \Sigma$. The boundary restriction is then, according to (2.5),

$$
\mathcal{R}_{\partial \Sigma}(\bar{\partial})=D_{\Sigma}^{+}(t)+\frac{1}{2} h=-i \partial_{\boldsymbol{e}_{1}}+\frac{1}{2} h .
$$

Let us observe that along the boundary we have $\partial_{\boldsymbol{e}_{1}}=\partial_{s}$. A simple computation shows that the mean curvature is the restriction to $t=0$ of the function $w_{t}^{\prime}$.

Consider the Atiyah-Patodi-Singer operator

$$
\bar{\partial}_{A P S}: \operatorname{Dom}\left(\bar{\partial}_{A P S}\right) \subset L^{2}(\Sigma) \rightarrow L^{2}(\Sigma), \quad \bar{\partial}_{A P S} u=\bar{\partial} u, \forall u \in \operatorname{Dom}\left(\bar{\partial}_{A P S}\right),
$$

where

$$
\operatorname{Dom}\left(\bar{\partial}_{A P S}\right)=\left\{u \in L^{1,2}(\Sigma, \mathbb{C}) ;\left.u\right|_{\partial \Sigma} \in \Lambda_{\partial}^{-}\right\}
$$


and $\Lambda_{\partial}^{-}$is the closed subspace of $L^{2}(\partial \Sigma)$ generated by the eigenvectors of the operator $B:=\mathcal{R}_{\partial \Sigma}(\bar{\partial})$ corresponding to strictly negative eigenvalues.

The index theorem of [8, 9] implies $\bar{\partial}_{A P S}$ is Fredholm and

$$
i_{A P S}(\Sigma, g):=\operatorname{index}\left(\bar{\partial}_{A P S}\right)=\frac{1}{2} \int_{\Sigma} c_{1}(\Sigma, g)-\xi_{B}, \xi_{B}:=\frac{1}{2}\left(\operatorname{dim} B+\eta_{B}(0)\right) .
$$

Above, $c_{1}(\Sigma, g) \in \Omega^{2}(\Sigma)$ is the 2 -form $\frac{1}{2 \pi} K_{g} d V_{g}$, where $K_{g}$ denotes the sectional curvature of $g$ and $d V_{g}$ denotes the metric volume form on $\Sigma$. From the GaussBonnet theorem for manifolds with boundary [15, §6.6] we deduce

$$
\int_{\Sigma} c_{1}(\Sigma, g)+\frac{1}{2 \pi} \int_{\partial \Sigma} h d s=\chi(\Sigma)
$$

where $h: \partial \Sigma \rightarrow \mathbb{R}$ is the mean curvature function defined as above. We deduce

$$
i_{A P S}(\Sigma, g)=\frac{1}{2} \chi(\Sigma)-\frac{1}{4 \pi} \int_{\Sigma} h d s-\xi_{B} .
$$

\section{Dolbeault operators on two-dimensional Cobordisms}

When thinking of cobordisms we adopt the Morse theoretic point of view. For us an elementary (nontrivial) 2-dimensional cobordism will be a pair $(\Sigma, f)$, where $\Sigma$ is a compact, connected, oriented surface with boundary and $f: \Sigma \rightarrow \mathbb{R}$ is a Morse function with a unique critical point $p_{0}$ located in the interior of $\Sigma$ such that

$$
f(\Sigma)=[-1,1], \quad f(\partial \Sigma)=\{-1,1\}, \quad f\left(p_{0}\right)=0 .
$$

In more intuitive terms, an elementary cobordism looks like one of the two pairs of pants in Figure 1, where the Morse function is understood to be the altitude.
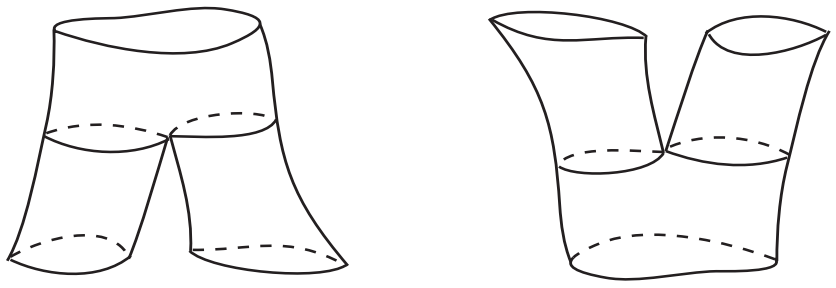

FIGURE 1. Elementary 2-dimensional cobordisms.

We set

$$
\partial_{ \pm} \Sigma:=f^{-1}( \pm 1)
$$

In the sequel, for simplicity, we will assume that $\partial_{+} \Sigma$ is connected, i.e., the pair $(\Sigma, f)$ looks like the left-hand side of Figure 1 .

We fix a Riemann metric $g$ on $\Sigma$. For simplicity 6 we assume that in an open neighborhood $\mathcal{O}$ near $p_{0}$ there exist local coordinates such that, in these coordinates, we have

$$
g=d x^{2}+d y^{2}, \quad f(x, y)=-\alpha x^{2}+\beta y^{2},
$$

where $\alpha, \beta$ are positive constants. We let $\nabla f$ denote the gradient of $f$ with respect to this metric and we set

$$
C_{t}:=f^{-1}(t), \quad t \neq 0
$$

${ }^{6}$ The results to follow do not require the simplifying assumption (3.1) but the computations would be less transparent. 
For $t \neq 0$ we regard $C_{t}$ as co-oriented by the gradient $\nabla f$. We let $h_{t}: C_{t} \rightarrow \mathbb{R}$ be the mean curvature of this co-oriented surface. For $t \neq 0$ we set

$$
L_{t}=\int_{C_{t}} d s=\operatorname{length}\left(C_{t}\right), \omega_{t}:=\frac{1}{4 \pi} \int_{C_{t}} h_{t} d s .
$$

The singular level set $C_{0}$ is also equipped with a natural measure defined by the arclength measure on $C_{0} \backslash\{0\}$. The length of $C_{0}$ is finite since in a neighborhood of the singular point $p_{0}$ the level set isometric to a pair of intersecting line segments in a Euclidean space.

Denote by $W^{ \pm}$the stable/unstable manifolds of $p_{0}$ with respect to the flow $\Phi^{t}$ generated by $-\nabla f$. The unstable manifold intersects the region $\{-1 \leq f<0\}$ in two smooth paths (see the bottom half of Figure 2)

$$
[-1,0) \ni t \mapsto a_{t}, b_{t} \in C_{t}, \quad \forall t \in[-1,0),
$$

while the stable manifold intersects the region $\{0<f \leq 1\}$ in two smooth paths (the top half of Figure 2)

$$
(0,1] \ni t \mapsto a_{t}, b_{t} \in C_{t}, \quad \forall t \in(0,1] .
$$

Observe that $\lim _{t \rightarrow 0} a_{t}=\lim _{t \rightarrow 0} b_{t}=p_{0}$. For this reason we set $a_{0}=b_{0}=p_{0}$.

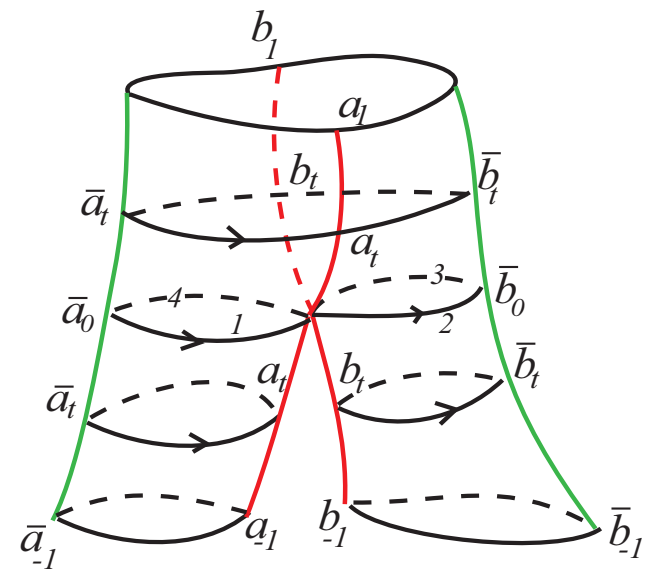

Figure 2. Cutting an elementary 2-dimensional cobordism.

As we have mentioned before, for $t<0$ the level set $C_{t}$ consists of two curves. We denote by $C_{t}^{a}$ the component containing the point $a_{t}$ and by $C_{t}^{b}$ the component containing $b_{t}$. For $t<0$ we set

$$
L_{t}^{a}:=\int_{C_{t}^{a}} d s, \quad L_{t}^{b}:=\int_{C_{t}^{b}} d s, \omega_{t}^{a}:=\frac{1}{4 \pi} \int_{C_{t}^{a}} h_{t} d s, \omega_{t}^{b}:=\frac{1}{4 \pi} \int_{C_{t}^{b}} h_{t} d s
$$

so that

$$
L_{t}=L_{t}^{a}+L_{t}^{b}, \quad \omega_{t}=\omega_{t}^{a}+\omega_{t}^{b}, \forall t<0 .
$$

Fix a point $\bar{a}_{-1} \in C_{-1}^{a} \backslash\left\{a_{-1}\right\}$ and a point $\bar{b}_{-1} \in C_{-1}^{b} \backslash\left\{b_{-1}\right\}$. For $t \in[-1,1]$ we denote by $\bar{a}_{t}$ (respectively $\bar{b}_{t}$ ) the intersection of $C_{t}$ with the negative gradient flow line through $\bar{a}_{-1}$ (respectively $\bar{b}_{-1}$ ). We obtain in this fashion two smooth maps $\bar{a}, \bar{b}:[-1,1] \rightarrow \Sigma$; see Figure 2, For $t>0$ we denote by $I_{t}^{a}$ the component 
of $C_{t} \backslash\left\{a_{t}, b_{t}\right\}$ that contains the point $\bar{a}_{t}$ and by $I_{t}^{b}$ the component of $C_{t} \backslash\left\{a_{t}, b_{t}\right\}$ that contains the point $\bar{b}_{t}$.

The regular part $C_{0}^{*}=C_{0} \backslash\left\{p_{0}\right\}$ consists of two components $C_{0}^{a}$ and $C_{0}^{b}$. We set

$$
\frac{1}{4 \pi} \omega_{0}^{a}:=\frac{1}{4 \pi} \int_{C_{0}^{a}} h_{0} d s, \omega_{0}^{b}:=\frac{1}{4 \pi} \int_{C_{0}^{b}} h_{0} d s, \omega_{0}:=\frac{1}{4 \pi} \int_{C_{0}^{*}} h_{0} d s=\omega_{0}^{a}+\omega_{0}^{b} .
$$

Note that the limits $\lim _{t \rightarrow 0} L_{t}^{a}, \lim _{t \rightarrow 0} L_{t}^{b}$ exist and are finite. We denote them by $L_{0}^{a}$ and respectively by $L_{0}^{b}$. We have

$$
L_{0}^{a}+L_{0}^{b}=L_{0}:=\text { length }(C)_{0} .
$$

Let $D_{t}$ denote the restriction of $\bar{\partial}$ to the co-oriented curve $C_{t}, t \neq 0$. As explained in the previous section we have

$$
D_{t}= \begin{cases}-\boldsymbol{i} \frac{d}{d s}+\frac{1}{2} h_{t}, & t>0, \\ \left.\left.\left(-i \frac{d}{d s}+\frac{1}{2} h_{t}\right)\right|_{C_{t}^{a}} \oplus\left(-\boldsymbol{i} \frac{d}{d s}+\frac{1}{2} h_{t}\right)\right|_{C_{t}^{b},} & t<0 .\end{cases}
$$

If we set

$$
\rho_{t}:=\omega_{t}^{a}-\left\lfloor\omega_{t}\right\rfloor, \quad \rho_{t}^{a}:=\omega_{t}^{b}-\left\lfloor\omega_{t}^{a}\right\rfloor, \quad \rho_{t}^{b}:=\omega_{t}^{b}-\left\lfloor\omega_{t}^{b}\right\rfloor,
$$

then the computations in Section 1 imply

$$
\xi(t):=\xi_{D_{t}}=\frac{1}{2} \begin{cases}1-2 \rho_{t}, & t>0 \\ \left(1-2 \rho_{t}^{a}\right)+\left(1-2 \rho_{t}^{b}\right), & t<0 .\end{cases}
$$

Throughout this and the next section we assume that both $D_{ \pm 1}$ are invertible.

We organize the family of complex Hilbert spaces $L^{2}\left(C_{t}, d s ; \mathbb{C}\right), t \in[-1,1]$ as a trivial bundle of Hilbert spaces as follows.

First, observe that $C_{0} \backslash\left\{\bar{a}_{0}, \bar{b}_{0}, p_{0}\right\}$ is a disjoint union of four open $\operatorname{arcs} I_{1}, \ldots, I_{4}$ labeled as in Figure 2, Denote by $\ell_{j}$ the length of $I_{j}$ so that

$$
L_{0}=\ell_{1}+\cdots+\ell_{4}, \quad L_{0}^{a}=\ell_{1}+\ell_{4}, \quad L_{0}^{b}=\ell_{2}+\ell_{3} .
$$

For $t>0$ we can isometrically identify the oriented open $\operatorname{arc} C_{t} \backslash \bar{a}_{t}$ with the open interval $\left(0, L_{t}\right)$. In this fashion we obtain a canonical isomorphism

$$
\mathcal{J}_{t}^{+}:=L^{2}\left(C_{t}, d s ; \mathbb{C}\right) \rightarrow L^{2}\left(\left[0, L_{t}\right] ; \mathbb{C}\right) .
$$

The rescaling $\left(0, L_{0}\right) \ni t \mapsto t / \lambda_{t} \in\left(0, L_{t}\right), \lambda_{t}=L_{0} / L_{t}$, induces as in Remark 1.6] a Hilbert space isomorphism

$$
\mathcal{R}_{t}^{+}: L^{2}\left(\left[0, L_{t}\right] ; \mathbb{C}\right) \rightarrow L^{2}\left(\left[0, L_{0}\right] ; \mathbb{C}\right)=: \boldsymbol{H}_{0} .
$$

Note that we have a partition $\mathcal{P}_{+}$of $\left[0, L_{0}\right]$,

$$
0=t_{0}<t_{1}<t_{2}<t_{3}<t_{4}=L_{0}, t_{j}-t_{j-1}=\ell_{j}, \forall j=1, \ldots, 4 .
$$

In this notation, the points corresponding to $t_{1}$ and $t_{3}$ belong to the stable manifold of the critical point $p_{0}$. This defines a Hilbert space isomorphism

$$
\mathcal{U}_{+}: L^{2}\left(\left[0, L_{0}\right] ; \mathbb{C}\right) \rightarrow \bigoplus_{j=1}^{4} L^{2}\left(\left[t_{j-1}, t_{j}\right] ; \mathbb{C}\right)=\bigoplus_{j=1}^{4} L^{2}\left(I_{j}, d s ; \mathbb{C}\right)=: \boldsymbol{H}_{0} .
$$

For $t<0$ we have

$$
L^{2}\left(C_{t}, d s ; \mathbb{C}\right)=L^{2}\left(C_{t}^{a}, d s ; \mathbb{C}\right) \oplus L^{2}\left(C_{t}^{b}, d s ; \mathbb{C}\right) .
$$

By removing the points $\bar{a}_{t}$ and $\bar{b}_{t}$ we obtain Hilbert space isomorphisms

$$
L^{2}\left(C_{t}^{a}, d s ; \mathbb{C}\right) \rightarrow L^{2}\left(\left[0, L_{t}^{a}\right] ; \mathbb{C}\right), L^{2}\left(C_{t}^{b}, d s ; \mathbb{C}\right) \rightarrow L^{2}\left(\left[0, L_{t}^{b}\right] ; \mathbb{C}\right)
$$


that add up to a Hilbert space isomorphism

$$
\mathcal{J}_{t}^{-}: L^{2}\left(C_{t}, d s ; \mathbb{C}\right) \rightarrow L^{2}\left(\left[0, L_{t}^{a}\right] ; \mathbb{C}\right) \oplus L^{2}\left(\left[0, L_{t}^{b}\right] ; \mathbb{C}\right) .
$$

By rescaling we obtain a Hilbert space isomorphism

$$
\mathcal{R}_{t}^{-}: L^{2}\left(\left[0, L_{t}^{a}\right] ; \mathbb{C}\right) \oplus L^{2}\left(\left[0, L_{t}^{b}\right] ; \mathbb{C}\right) \rightarrow L^{2}\left(\left[0, L_{0}^{a}\right] ; \mathbb{C}\right) \oplus L^{2}\left(\left[0, L_{0}^{b}\right] ; \mathbb{C}\right) .
$$

Next observe that we have isomorphisms

$$
\begin{aligned}
& \mathcal{U}_{-}^{a}: L^{2}\left(\left[0, L_{0}^{a}\right] ; \mathbb{C}\right) \rightarrow L^{2}\left(I_{1}, d s ; \mathbb{C}\right) \oplus L^{2}\left(I_{4}, d s \mathbb{C}\right), \\
& \mathcal{U}_{-}^{b}: L^{2}\left(\left[0, L_{0}^{b}\right] ; \mathbb{C}\right) \cong L^{2}\left(I_{3}, d s ; \mathbb{C}\right) \oplus L^{2}\left(I_{3}, d s ; \mathbb{C}\right)
\end{aligned}
$$

that add up to an isomorphism

$$
\mathcal{U}_{-}: L^{2}\left(\left[0, L_{0}\right] ; \mathbb{C}\right) \rightarrow \bigoplus_{j=1}^{4} L^{2}\left(I_{j}, d s ; \mathbb{C}\right) .
$$

For $t=0$ we let $\mathcal{J}_{0}$ be the natural isomorphism

$$
\jmath_{0}: L^{2}\left(C_{0}, d s ; \mathbb{C}\right) \rightarrow \bigoplus_{j=1}^{4} L^{2}\left(I_{j}, d s ; \mathbb{C}\right) \cong \boldsymbol{H}_{0} .
$$

Now define

$$
\mathcal{J}_{t}:= \begin{cases}\mathcal{U}_{+} \mathcal{R}_{t}^{+} \mathcal{J}_{t}^{+}, & t>0, \\ \mathcal{U}_{-} \mathcal{R}_{t}^{-} \mathcal{J}_{t}^{-}, & t<0, \\ \mathcal{J}_{0}, & t=0 .\end{cases}
$$

We use that the collection of isomorphisms $\mathcal{J}_{t}$ organizes the collection $L^{2}\left(C_{t}, d s ; \mathbb{C}\right)$ as a trivial Hilbert $\mathcal{H}$-bundle over $[-1,1]$.

Theorem 3.1. (a) The operators $\mathcal{D}_{t}:=\mathcal{J}_{t} D_{t} \mathcal{J}_{t}^{-1}$ converge in the gap topology as $t \rightarrow 0^{ \pm}$to Fredholm, selfadjoint operators $\mathcal{D}_{0}^{ \pm}$.

(b) The eta invariants of $\mathcal{D}_{0}^{ \pm}$exist, and we set

$$
\xi_{ \pm}:=\frac{1}{2}\left(\operatorname{dim} \operatorname{ker} \mathcal{D}_{0}^{ \pm}+\eta_{\mathcal{D}_{0}^{ \pm}}(0)\right) .
$$

If $\operatorname{ker} \mathcal{D}_{0}^{ \pm}=0$, then we have 7

$$
i_{A P S}(\bar{\partial})+\lim _{\varepsilon \rightarrow 0^{+}}\left(S F\left(\mathcal{D}_{t} ; \varepsilon<t \leq 1\right)+S F\left(\mathcal{D}_{t},-1 \leq t<-\varepsilon\right)\right)=-\left(\xi_{+}-\xi_{-}\right) .
$$

Proof. We set

$$
\mathcal{S}_{t}:= \begin{cases}\mathcal{U}_{+}^{-1} \mathcal{D}_{t} \mathcal{U}_{+}, & t>0 \\ \mathcal{U}_{-}^{-1} \mathcal{D}_{t} \mathcal{U}_{-}, & t<0 .\end{cases}
$$

To establish the convergence statements we show that the $\operatorname{limits} \lim _{t \rightarrow 0^{ \pm}} \mathcal{S}_{t}$ exist in the gap topology of the space of unbounded selfadjoint operators on $L^{2}\left(0, L_{0} ; \mathbb{C}\right)$. We discuss separately the cases $\pm t>0$, corresponding to restrictions to level sets above/below the critical level set $\{f=0\}$.

\footnotetext{
${ }^{7}$ The condition $\operatorname{ker} \mathcal{D}_{0}^{ \pm}=0$ is satisfied for an open and dense set of metrics $g$ satisfying (3.1). When this condition is violated the identity (3.5) needs to be slightly modified to take into account these kernels.
} 
A. $t>0$. We observe that

$$
\operatorname{Dom}\left(\mathcal{S}_{t}\right)=\left\{u \in L^{1,2}\left(0, L_{0} ; \mathbb{C}\right) ; u\left(L_{0}\right)=u(0)\right\}, \quad \mathcal{S}_{t}(u)=-i \lambda_{t} \frac{d}{d s}+\frac{1}{2} h_{t}\left(s / \lambda_{t}\right),
$$

where we recall that the constant $\lambda_{t}$ is the rescaling factor $L_{0} / L_{t}$. We set

$$
K_{t}(s):=\frac{1}{\lambda_{t}} \int_{0}^{s} h_{t}\left(\sigma / \lambda_{t}\right) d \sigma .
$$

Using the fact that $\lambda_{t} \rightarrow 1$ and Proposition 1.3 we see that it suffices to show that $K_{t}$ is very weakly convergent in $\mathcal{A}_{L_{0}}$; see Definition 1.1. Thus it suffices to prove two things:

$\left(\mathbf{A}_{1}\right)$

The limit $\lim _{t \rightarrow 0^{+}} K_{t}\left(L_{0}\right)$ exists.

$\left(\mathbf{A}_{\mathbf{2}}\right) \quad$ The limits $\lim _{t \rightarrow 0^{+}} K_{t}(s)$ exist for almost any $s \in\left(0, L_{0}\right)$.

Proof of $\mathbf{\mathbf { A } _ { \mathbf { 1 } }}$. Observe that

$$
K_{t}\left(L_{0}\right)=\int_{C_{t}} h_{t} d s=\int_{C_{t}-\mathcal{O}} h_{t} d s+\int_{\mathcal{O} \cap C_{t}} h_{t} d s,
$$

where $\mathcal{O}$ is the neighborhood where (3.1) holds. The intersection of $C_{t}$ with $\mathcal{O}$ is depicted in Figure 3 .

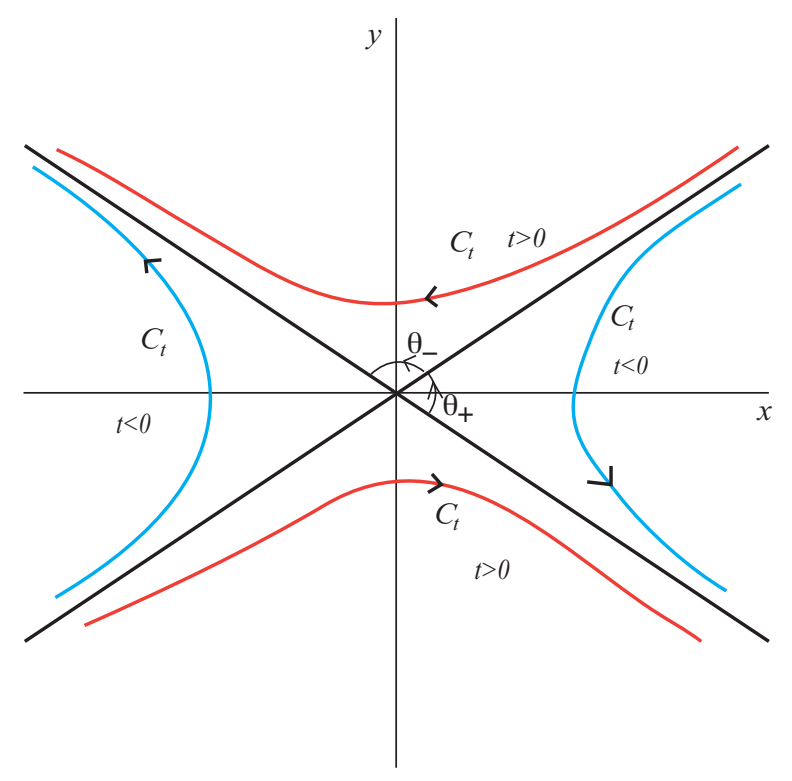

FiguRE 3 . The behavior of $C_{t}$ near the critical point.

The integral $\int_{C_{t} \backslash \mathcal{O}} h_{t} d s$ converges as $t \rightarrow 0^{+}$to $\int_{C_{0} \backslash \mathcal{O}} h_{0} d s$. Next, observe that the intersection $C_{t} \cap \mathcal{O}$ consists of two oriented arcs (see Figure 3) and the integral $\int_{\mathcal{O}^{\prime} C_{t}} h_{t}$ computes the total angular variation of the oriented unit normal vector field along these oriented arcs. (The orientation of the normal is given by the 
gradient of the Morse function.) Using the notation in Figure 3 we see that this total variation approaches $-2 \theta_{+}$as $t \rightarrow 0+$. Hence

$$
\lim _{t \rightarrow 0^{+}} K_{t}\left(L_{0}\right)=\int_{C_{0} \backslash 0} h_{0} d s-2 \theta_{+}
$$

so that

$$
\omega_{0}^{+}=\lim _{t \rightarrow 0^{+}} \omega_{t}=\frac{1}{4 \pi} \lim _{t \rightarrow 0^{+}} \int_{C_{t}} h_{t} d s=\omega_{0}-\frac{\theta_{+}}{2 \pi} .
$$

Proof of $\left(\mathbf{A}_{\mathbf{2}}\right.$. Let $C_{t}^{*}:=C_{t} \backslash\left\{\bar{a}_{t}\right\}$ and define $s=s(q): C_{t}^{*} \rightarrow(0, \infty)$ to be the coordinate function on $C_{t}^{*}$ such that the resulting map $C_{t}^{*} \ni q \mapsto \sigma(q)=s(q) / \lambda_{t} \in \mathbb{R}$ is an orientation-preserving isometry onto $\left(0, L_{t}\right)$. In other words, $\sigma$ is the oriented arclength function measured starting at $\bar{a}_{t}$, and $s$ defines a diffeomorphism $C_{t}^{*} \rightarrow\left(0, L_{0}\right)$. Let $q_{t}:\left(0, L_{0}\right) \rightarrow C_{t}^{*}$ be the inverse of this diffeomorphism.

Consider the partition (3.4). Observe that there exist positive constants $c$ and $\varepsilon$ such that, whenever

$$
\forall t \in(0, \varepsilon), \quad \forall s \in\left[t_{1}-c, t_{1}+c\right] \cup\left[t_{3}-c, t_{3}+c\right]: q_{t}(s) \in \mathcal{O},
$$

the numbers $t_{j}$ are defined by (3.4). Intuitively, the intervals $\left[t_{1}-c, t_{1}+c\right] \cup\left[t_{3}-\right.$ $\left.c, t_{3}+c\right]$ collect the parts of $C_{t}$ that are close to the critical point $p_{0}$. The lengths of each of the two components of $C_{t}$ that are close to $p_{0}$ are bounded from below by $2 c / \lambda_{t}$.

To prove part (b) it suffices to understand the behavior of $K_{t}(s)$ for $s \in\left[t_{1}-\right.$ $\left.c, t_{1}+c\right] \cup\left[t_{3}-c, t_{3}+c\right]$. We do this for one of the components since the behavior for the other component is entirely similar. We look at the component of $C_{t} \cap \mathcal{O}$ that lies in the lower half-plane in Figure 3 ,

Here is a geometric approach. As explained before, the difference $K_{t}(s)-K_{t}\left(t_{1}-\right.$ c) computes the angular variation of the oriented unit normal to $C_{t}$ over the interval $\left[t_{1}-c, s\right]$. A close look at Figure 3 shows that the absolute value of this is bounded above by $\theta_{+}$. This proves the boundedness part of the bounded convergence theorem. The almost everywhere convergence is also obvious in view of the above geometric interpretation. The limit function is a bounded function $K_{0}:\left[0, L_{0}\right] \rightarrow \mathbb{R}$ that has jumps $-\theta_{+}$at $t_{1}$ and $t_{3}$,

$$
K_{0}\left(t_{1}+0\right)-K_{0}\left(t_{1}-0\right)=K\left(t_{3}+0\right)-K\left(t_{3}-0\right)=-\theta_{+},
$$

while the continuous function

$$
K_{0}(t)+\theta_{+} H\left(t-t_{1}\right)+\theta_{+} H\left(t-t_{3}\right)
$$

is differentiable everywhere on $\left[0, L_{0}\right] \backslash\left\{t_{1}, t_{3}\right\}$ and the derivative is the mean curvature function $h_{0}$ of $C_{0} \backslash\left\{p_{0}\right\}$.

We can now invoke Theorem 1.8 to conclude that the operators $\mathcal{D}_{t}$ converge as $t \rightarrow 0^{+}$to the operator

$$
\mathcal{D}_{0}^{+}: \operatorname{Dom}\left(\mathcal{D}_{0}^{+}\right) \subset L^{2}\left(0, L_{0} ; \mathbb{C}\right) \rightarrow L^{2}\left(0, L_{0} ; \mathbb{C}\right),
$$


where $\operatorname{Dom}\left(\mathcal{D}_{0}^{+}\right)$consists of functions $u \in L^{2}\left(0, L_{0} ; \mathbb{C}\right)$ such that, if we denote by $u_{j}$ the restriction of $u$ to $I_{j}=\left(t_{j-1}, t_{j}\right), 1 \leq j \leq 4$, then

$$
\begin{aligned}
& u_{j} \in L^{1,2}\left(I_{j}\right), \forall j=1, \ldots, 4, \\
& \left\{\begin{array}{l}
\gamma_{-} u_{2}=e^{i \theta_{+} / 2} \gamma_{+} u_{1}, \\
\gamma_{-} u_{4}=e^{i \theta_{+} / 2} \gamma_{+} u_{3}, \\
\gamma_{+} u_{2}=\gamma_{-} u_{3}, \\
\gamma_{+} u_{+}=\gamma_{-} u_{0},
\end{array}\right.
\end{aligned}
$$

while for $u \in \operatorname{Dom}\left(\mathcal{D}_{0}^{+}\right)$we have

$$
\left.\left(\mathcal{D}_{0}^{+} u\right)\right|_{I_{j}}=\left(-\boldsymbol{i} \frac{d}{d s}+\frac{1}{2} h_{0}(s)\right) u_{j}, \quad \forall j=1, \ldots, 4 .
$$

Using the point of view elaborated upon in Remark 1.9, we let $I$ denote the disjoint union of the intervals $I_{j}, j=1, \ldots, 4$. We regard $\mathcal{D}_{0}^{+}$as a closed densely defined operator on the Hilbert space $L^{2}(I, \mathbb{C})$ with domain consisting of quadruples $\boldsymbol{u}=$ $\left(u_{1}, \ldots, u_{4}\right) \in L^{1,2}(I)$ satisfying the boundary condition

$$
\gamma_{-} \boldsymbol{u}=\boldsymbol{T}_{+} \gamma_{+} \boldsymbol{u}
$$

where

$$
\boldsymbol{T}_{+}: \mathbb{C}^{4} \cong L^{2}\left(\partial_{+} I\right) \rightarrow L^{2}\left(\partial_{-} I\right) \cong \mathbb{C}^{4}
$$

is the transmission operator given by the unitary $4 \times 4$ matrix

$$
\boldsymbol{T}_{+}=\left[\begin{array}{cccc}
0 & 0 & 0 & 1 \\
e^{i \theta_{+} / 2} & 0 & 0 & 0 \\
0 & 1 & 0 & 0 \\
0 & 0 & e^{i \theta_{+} / 2} & 0
\end{array}\right] \text { and } \mathcal{D}_{0}^{+}\left[\begin{array}{c}
u_{1} \\
\vdots \\
u_{4}
\end{array}\right]=\left(-\boldsymbol{i} \frac{d}{d s}+\frac{1}{2} h_{0}\right)\left[\begin{array}{c}
u_{1} \\
\vdots \\
u_{4}
\end{array}\right]
$$

Using (1.10) we deduce that

$$
\xi^{+}=\xi_{\mathcal{D}_{0}^{+}}=\frac{1}{2}\left(1-2 \rho_{+}\right), \quad \rho_{+}=\omega_{0}^{+}-\left\lfloor\omega_{0}^{+}\right\rfloor=\omega_{0}-\frac{\theta_{+}}{2 \pi}-\left\lfloor\omega_{0}-\frac{\theta_{+}}{2 \pi}\right\rfloor .
$$

B. $t<0$. We observe that $\mathcal{S}_{t}=\mathcal{S}_{t}^{a} \oplus \mathcal{S}_{t}^{b}$, where for $\bullet=a, b$ we have

$$
\mathcal{S}_{t}^{\bullet}: \operatorname{Dom}\left(\mathcal{S}_{t}^{\bullet}\right) \subset L^{2}\left(0, L_{0}^{\bullet} ; \mathbb{C}\right) \rightarrow L^{2}\left(0, L_{0}^{\bullet} ; \mathbb{C}\right)
$$

$\operatorname{Dom}\left(\mathcal{S}_{t}^{\bullet}\right)=\left\{u \in L^{1,2}\left(0, L_{0}^{\bullet} ; \mathbb{C}\right) ; u\left(L_{0}^{\bullet}\right)=u(0)\right\}, \quad \mathcal{S}_{t}^{\bullet} u=-\boldsymbol{i} \lambda_{t}^{\bullet} \frac{d}{d s}+\frac{1}{2} h_{t}\left(s / \lambda_{t}^{\bullet}\right)$,

and $\lambda_{t}^{\bullet}$ is the rescaling factor $\frac{L_{0}^{\bullet}}{L_{t}^{\bullet}}$. It is convenient to regard $\mathcal{S}_{t}^{\bullet}$ as defined on the component $C_{0}^{\bullet}$ of $C_{0}^{*}$. Observe that $C_{0}^{a} \backslash\left\{\bar{a}_{0}\right\}=I_{1} \cup I_{4}$ and $C_{0}^{b} \backslash\left\{\bar{b}_{0}\right\}=I_{2} \cup I_{3}$. Arguing as in the case $t>0$ we conclude that

$$
\lim _{t \nearrow 0} \omega_{t}^{a}=\omega_{0}^{a}+\frac{\theta_{-}}{4 \pi}, \lim _{t \nearrow 0} \omega_{t}^{b}=\omega_{0}^{a}+\frac{\theta_{-}}{4 \pi}, \omega_{0}^{-}:=\lim _{t \nearrow 0} \omega_{t}=\omega_{0}+\frac{\theta_{-}}{2 \pi},
$$

and that the operators $\mathcal{D}_{t}^{a}$ and $\mathcal{D}_{t}^{b}$ converge in the gap topology as $t \rightarrow 0^{-}$to the operators

$$
\begin{aligned}
& \mathcal{D}_{0}^{a}: \operatorname{Dom}\left(\mathcal{D}_{0}^{a}\right) \subset L^{2}\left(I_{1}\right) \oplus L^{2}\left(I_{4}\right) \rightarrow L^{2}\left(I_{1}\right) \oplus L^{2}\left(I_{4}\right), \\
& \mathcal{D}_{0}^{b}: \operatorname{Dom}\left(\mathcal{D}_{0}^{b}\right) \subset L^{2}\left(I_{2}\right) \oplus L^{2}\left(I_{3}\right) \rightarrow L^{2}\left(I_{2}\right) \oplus L^{2}\left(I_{3}\right),
\end{aligned}
$$

where $\operatorname{Dom}\left(\mathcal{D}_{0}^{a}\right)$ consists of functions $\left(u_{1}, u_{4}\right) \in L^{1,2}\left(I_{1}\right) \oplus L^{1,2}\left(I_{4}\right)$ such that

$$
\gamma_{-} u_{4}=e^{-i \theta_{-} / 2} \gamma_{+} u_{1}, \quad \gamma_{+} u_{4}=\gamma_{-} u_{1}
$$


$\operatorname{Dom}\left(\mathcal{D}_{0}^{b}\right)$ consists of functions $\left(u_{2}, u_{3}\right) \in L^{1,2}\left(I_{3}\right) \oplus L^{1,2}\left(I_{3}\right)$ such that

$$
\gamma_{-} u_{2}=e^{-i \theta_{-} / 4 \pi} \gamma_{+} u_{3}, \quad \gamma_{-} u_{3}=\gamma_{+} u_{2},
$$

where $\theta_{-}$is depicted in Figure 3, and

$$
\begin{aligned}
& \mathcal{D}_{0}^{a}\left(u_{1}, u_{4}\right)=\left(-i \frac{d u_{1}}{d s}+\frac{1}{2} h_{0} u_{1},-i \frac{d u_{4}}{d s}+\frac{1}{2} h_{0} u_{4}\right), \\
& \mathcal{D}_{0}^{a}\left(u_{2}, u_{3}\right)=\left(-i \frac{d u_{2}}{d s}+\frac{1}{2} h_{0} u_{2},-i \frac{d u_{3}}{d s}+\frac{1}{2} h_{0} u_{3}\right) .
\end{aligned}
$$

The direct sum $\mathcal{D}_{0}^{-}=\mathcal{D}_{0}^{a} \oplus \mathcal{D}_{0}^{b}$ is the closed densely defined linear operator on $L^{2}(I)$ with domain of quadruples $\boldsymbol{u}=\left(u_{1}, \ldots, u_{4}\right) \in L^{1,2}(I, \mathbb{C})$ satisfying the boundary condition

$$
\gamma_{-} \boldsymbol{u}=\boldsymbol{T}_{-} \gamma_{+} \boldsymbol{u}
$$

where $\boldsymbol{T}_{-}: \mathbb{C}^{4} \cong L^{2}\left(\partial_{+} I\right) \rightarrow L^{2}\left(\partial_{+} I\right) \cong \mathbb{C}^{4}$ is the transmission operator given by the unitary $4 \times 4$ matrix

$\boldsymbol{T}_{-}=\left[\begin{array}{cccc}0 & 0 & 0 & 1 \\ 0 & 0 & e^{-i \theta_{-} / 2} & 0 \\ 0 & 1 & 0 & 0 \\ e^{-i \theta_{-} / 2} & 0 & 0 & 0\end{array}\right]$ and $\mathcal{D}_{0}^{-}\left[\begin{array}{c}u_{1} \\ \vdots \\ u_{4}\end{array}\right]=\left(-\boldsymbol{i} \frac{d}{d s}+\frac{1}{2} h_{0}\right)\left[\begin{array}{c}u_{1} \\ \vdots \\ u_{4}\end{array}\right]$.

Then $\xi_{-}=\xi_{-}^{a}+\xi_{-}^{b}$, where for $\bullet=a, b$ we have

$$
\xi_{-}^{\bullet}=\frac{1}{2}\left(1-2 \rho_{-}^{\bullet}\right), \quad \rho_{-}^{\bullet}=\omega_{0}^{\bullet}+\frac{\theta_{-}}{4 \pi}-\left\lfloor\omega_{0}^{\bullet}+\frac{\theta_{-}}{4 \pi}\right\rfloor .
$$

Combining (3.6) and (3.9) with the equality $\theta_{+}+\theta_{-}=\pi$ we deduce

$$
\omega_{0}^{+}-\omega_{0}^{-}=\lim _{t \searrow 0} \omega_{t}-\lim _{t \nearrow 0} \omega_{t}=-\frac{1}{2} .
$$

To prove (3.5) we use the index formula (2.8). We have

$$
\begin{gathered}
i_{A P S}(\bar{\partial})=-\frac{1}{2}-\omega_{1}+\omega_{-1}-\xi_{D_{1}}+\xi_{D_{-1}} \\
\stackrel{3.11}{=} \omega_{0}^{+}-\omega_{0}^{-}-\omega_{1}+\omega_{-1}-\xi_{D_{1}}+\xi_{D_{-1}} \\
=\left(\omega_{0}^{+}+\xi^{+}\right)-\left(\omega_{1}+\xi_{D_{1}}\right)-\left(\omega_{0}^{-}+\xi^{-}\right)+\left(\omega_{-1}+\xi_{D_{-1}}\right)-\left(\xi^{+}-\xi^{-}\right) \\
\stackrel{1.12}{=}-\lim _{\varepsilon \rightarrow 0^{+}} S F\left(\mathcal{D}_{t} ; \varepsilon<t \leq 1\right)-\lim _{\varepsilon \rightarrow 0^{+}} S F\left(\mathcal{D}_{t},-1 \leq t<-\varepsilon\right)-\left(\xi_{+}-\xi_{-}\right) .
\end{gathered}
$$

Remark 3.2. (a) We want to outline an analytic argument proving $\mathbf{\mathbf { A } _ { 2 }}$. Using (3.1) we deduce that this component has a parametrization compatible with the orientation given by

$$
y_{t}=-\left(\zeta_{t}+m x^{2}\right)^{1 / 2}, \quad|x|<d_{t},
$$

where $\zeta_{t}=\frac{t}{\beta}, m=\frac{\alpha}{\beta}$ and $d_{t}$ is such that the length of this arc is $2 c / \lambda_{t}$. Observe that there exists $d_{*}>0$ such that $\lim _{t \rightarrow 0^{+}} d t=d_{*}$. We have

$$
d y_{t}=-m x\left(\zeta_{t}+m x^{2}\right)^{-1 / 2} d x
$$


Set

$$
\begin{gathered}
y_{t}^{\prime}:=\frac{d y_{t}}{d x}=-m x\left(\zeta_{t}+m x^{2}\right)^{-1 / 2} \\
y_{t}^{\prime \prime}:=\frac{d^{2} y_{t}}{d x^{2}}=-m\left(\zeta_{t}+m x^{2}\right)^{-1 / 2}+m^{2} x^{2}\left(\zeta_{t}+m x^{2}\right)^{-3 / 2}=-\frac{m \zeta_{t}}{\left(\zeta_{t}+m x^{2}\right)^{3 / 2}} .
\end{gathered}
$$

The arclength is

$$
d \sigma^{2}=\left(1+\left(y_{t}^{\prime}\right)^{2}\right) d x^{2}=\underbrace{\left(1+\frac{m^{2} x^{2}}{\zeta_{t}+m x^{2}}\right)}_{=: w(t, x)^{2}} d x^{2} .
$$

The mean curvature $h_{t}$ is found using the Frenet formulæ, [15. More precisely, $h_{t}(x)=\frac{y_{t}^{\prime \prime}}{w^{3}}$. Then

$$
h_{t} d \sigma=h_{t} w d x=\frac{y_{t}^{\prime \prime}}{1+\left(y_{t}^{\prime}\right)^{2}} d x=-\frac{m \zeta_{t} d x}{\left(\zeta_{t}+m x^{2}\right)^{1 / 2}\left(\zeta_{t}+m x^{2}+m^{2} x^{2}\right)} .
$$

We now observe that we can write $h_{t} d \sigma=\phi_{t}^{*}\left(\rho_{\infty} d u\right)$, where $\phi_{t}$ is the rescaling map

$$
x \mapsto u=t^{-1 / 2} x \text { and } \rho_{\infty}(u)=-\frac{m \zeta_{1}}{\left(\zeta_{1}+m u^{2}\right)^{1 / 2}\left(\zeta_{1}+m u^{2}+m^{2} u^{2}\right)} .
$$

This allows us to conclude via a standard argument that the densities $h_{t} d \sigma$ converge very weakly as $t \rightarrow 0^{+}$to a $\delta$-measure concentrated at the origin.

(b) The results in Theorem 3.1 extend without difficulty to Dolbeault operators twisted by line bundles. More precisely, given a Hermitian line bundle $L$ and a Hermitian connection $A$ on $L$, we can form a Dolbeault operator $\bar{\partial}_{A}: C^{\infty}(L) \rightarrow$ $C^{\infty}\left(L \otimes K_{\Sigma}^{-1}\right)$. Fortunately, all the line bundles on the two-dimensional cobordism $\Sigma$ are trivializable. We fix a trivialization so that the connection $A$ can be identified with a purely imaginary 1 -form $A=i a, a \in \Omega^{1}(\Sigma)$. Then

$$
\bar{\partial}_{A}=\bar{\partial}+i a^{0,1} .
$$

The restriction of $D_{A}^{+}=\sqrt{2} \bar{\partial}_{A}$ to the co-oriented curve $C_{t}$ is

$$
D_{A}(t)=-i \nabla_{s}^{A}+\frac{1}{2} h_{t}=-i \frac{d}{d s}+\frac{1}{2} h_{t}+a_{t}, \quad a_{t}:=a\left(\frac{d}{d s}\right) \in \Omega^{0}\left(C_{t}\right) .
$$

As in the proof of Theorem 3.1 we only need to understand the behavior of $a_{t}$ in the neighborhood $\mathcal{O} \cap C_{t}$. Supposing for simplicity that $t>0$, we concentrate only on the component of $C_{t} \cap \mathcal{O}$ that lies in the lower half-plane of Figure 3, In the neighborhood $\mathcal{O}$ we can write

$$
a=p d x+q d y, \quad p, q \in C^{\infty}(\mathcal{O}) .
$$

Using the parametrization (3.12) we deduce that

$$
\left.a\right|_{C_{t} \cap \mathcal{O}}=\left(p-m q x\left(\zeta_{t}+m x^{2}\right)^{-1 / 2}\right) d x=a_{t} d s=a_{t} w d x .
$$

Hence, as $t \rightarrow 0^{+}$, the measure $a_{t} d s$ converges to the measure

$$
\left(p-m^{1 / 2}(2 H(x)-1)\right) d x \text {. }
$$


Remark 3.3. One may ask what happens in the case of a cobordism corresponding to a local min/max of a Morse function. In this case $\Sigma$ is a disk, the regular level sets $C_{t}$ are circles and the singular level set is a point. Consider for example the case of a local minimum. Assume that the metric near the minimum $p_{0}$ is Euclidean, and in some Euclidean coordinates near $p_{0}$ we have $f=x^{2}+y^{2}$. Then $C_{t}$ is the Euclidean circle of radius $t^{1 / 2}$, and the function $h_{t}$ is the constant function $h_{t}=t^{-1 / 2}$. Then $\omega_{t}=\frac{1}{2}, \xi_{t}=\frac{1}{2}$ and the Atiyah-Patodi-Singer index of $\bar{\partial}$ on the Euclidean disk of radius $t^{1 / 2}$ is 0 . The operator $D_{t}$ can be identified with the operator

$$
-i \frac{d}{d s}+\frac{1}{2 t^{1 / 2}}
$$

with periodic boundary conditions on the interval $\left[0,2 \pi t^{1 / 2}\right]$. Using the rescaling trick in Remark 1.6 we see that this operator is conjugate to the operator $L_{t}=$ $-t^{1 / 2} \boldsymbol{i} \frac{d}{d s}+\frac{1}{2}$ on the interval $[0,2 \pi]$ with periodic boundary conditions. The switched graphs of these operators,

$$
\begin{gathered}
\widetilde{\Gamma}_{L_{t}}=\left\{\left(L_{t} u, u\right) ; u \in L^{1,2}([0,2 \pi] ; \mathbb{C}) ; u(0)=u(2 \pi)\right\} \subset \boldsymbol{H} \oplus \boldsymbol{H}, \\
\boldsymbol{H}=L^{2}([0,2 \pi] ; \mathbb{C}),
\end{gathered}
$$

converge in the gap topology to the subspace $\boldsymbol{H}_{+}=\boldsymbol{H} \oplus 0 \subset \boldsymbol{H} \oplus \boldsymbol{H}$. This limit is not the switched graph of any operator. However, this limiting space forms a Fredholm pair with $\boldsymbol{H}_{-}=0 \oplus \boldsymbol{H}$ and invoking the results in [5] we conclude that

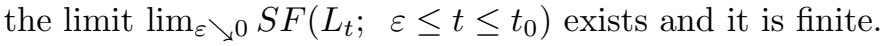

\section{The Kashiwara-Wall IndeX}

In this final section we would like to identify the correction term on the righthand side of (3.5) with a symplectic invariant that often appears in surgery formulæ. To this aim, we need to elaborate on the symplectic point of view first outlined in Remark 1.9

Fix a finite-dimensional complex Hermitian space $\boldsymbol{E}$, set $n:=\operatorname{dim}_{\mathbb{C}} \boldsymbol{E}$,

$$
\widehat{\boldsymbol{E}}:=\boldsymbol{E} \oplus \boldsymbol{E}, \quad \boldsymbol{E}_{+}:=\boldsymbol{E} \oplus 0, \quad \boldsymbol{E}_{-}:=0 \oplus \boldsymbol{E},
$$

and let $J: \widehat{\boldsymbol{E}} \rightarrow \widehat{\boldsymbol{E}}$ be the unitary operator given by the block decomposition

$$
J=\left[\begin{array}{cc}
-i & 0 \\
0 & i
\end{array}\right]
$$

We let $\boldsymbol{L} \boldsymbol{a g}$ denote the space of Hermitian Lagrangians on $\widehat{\boldsymbol{E}}$, i.e., complex subspaces $L \subset \widehat{\boldsymbol{E}}$ such that $L^{\perp}=J L$. As explained in [5, 14, any such Lagrangian can be identified with the graph 8 of a complex isometry $T: \boldsymbol{E}_{+} \rightarrow \boldsymbol{E}_{-}$, or equivalently, with the group $U(\boldsymbol{E})$ of unitary operators on $\boldsymbol{E}$. In other words, the graph map

$$
\Gamma: U(\boldsymbol{E}) \rightarrow \boldsymbol{L a g}(\widehat{\boldsymbol{E}}), \quad U(\boldsymbol{E}) \mapsto \Gamma_{T} \subset \widehat{\boldsymbol{E}}
$$

is a diffeomorphism. The involution $L \leftrightarrow J L$ on $\mathbf{L a g}$ corresponds via this diffeomorphism to the involution $T \leftrightarrow-T$ on $U(\boldsymbol{E})$.

\footnotetext{
${ }^{8}$ In [11, a Lagrangian is identified with the graph of an isometry $\boldsymbol{E}_{-} \rightarrow \boldsymbol{E}_{+}$, which explains why our formulæ will look a bit different from the ones in [11. Our choice is based on the conventions in [5], which seem to minimize the number of signs in the Schubert calculus on $\mathbf{L a g}$.
} 
We define a branch of the logarithm $\log : \mathbb{C}^{*} \rightarrow \mathbb{C}$ by requiring that $\mathbf{I m} \log \in$ $[-\pi, \pi)$. Equivalently,

$$
\log z=\int_{\gamma_{z}} \frac{d \zeta}{\zeta}
$$

where $\gamma_{z}:[0,1] \rightarrow \mathbb{C}$ is any smooth path from 1 to $z$ such that $\gamma_{z}(t) \notin(-\infty, 0]$, $\forall t \in[0,1)$. In particular, $\log (-1)=-\pi \boldsymbol{i}$. Following [11, §6] we define

$$
\tau: U(\boldsymbol{E}) \times U(\boldsymbol{E}) \rightarrow \mathbb{R}, \quad \tau\left(T_{0}, T_{1}\right)=\frac{1}{2 \pi \boldsymbol{i}} \operatorname{tr} \log \left(T_{1}^{-1} T_{0}\right)=\frac{1}{2 \pi \boldsymbol{i}} \sum_{\lambda \in \mathbb{C}^{*}}(\log \lambda) m_{\lambda},
$$

where $m_{\lambda}:=\operatorname{dim} \operatorname{ker}\left(\lambda-T_{1}^{-1} T_{0}\right)$. Observe that

$$
\begin{gathered}
e^{2 \pi \boldsymbol{i} \tau\left(T_{0}, T_{1}\right)}=\frac{\operatorname{det} T_{0}}{\operatorname{det} T_{1}}, \\
\tau\left(T_{0}, T_{1}\right)+\tau\left(T_{1}, T_{0}\right)=-\operatorname{dim} \operatorname{ker}\left(T_{0}+T_{1}\right) .
\end{gathered}
$$

Via the graph diffeomorphism we obtain a map

$$
\mu=\tau \circ \Gamma: \mathbf{L a g} \times \mathbf{L a g} \rightarrow \mathbb{R} .
$$

The equality (4.1b) can be rewritten as

$$
\tau\left(L_{0}, L_{1}\right)+\tau\left(L_{1}, L_{0}\right)=-\operatorname{dim}\left(L_{0} \cap J L_{1}\right)=-\operatorname{dim}\left(J L_{0} \cap L_{1}\right) .
$$

We want to relate the invariant $\tau$ to the eta invariant of a natural selfadjoint operator. We associate to each pair $L_{0}, L_{1} \in \boldsymbol{L} \boldsymbol{a g}$ the selfadjoint operator

$$
D_{L_{0}, L_{1}}: V\left(L_{0}, L_{1}\right) \subset L^{2}(I, \widehat{\boldsymbol{E}}) \rightarrow L^{2}(I, \widehat{\boldsymbol{E}}),
$$

where

$$
V\left(L_{0}, L_{1}\right)=\left\{u \in L^{1,2}(I, \widehat{\boldsymbol{E}}) ; u(0) \in L_{0}, u(1) \in L_{1}\right\}, \quad D_{L_{0}, L_{1}} u=J \frac{d u}{d t} .
$$

This is a selfadjoint operator with compact resolvent. We want to describe its spectrum, and in particular, prove that it has a well-defined eta invariant. Let $T_{0}, T_{1}: \boldsymbol{E}_{+} \rightarrow \boldsymbol{E}_{-}$denote the isometries associated to $L_{0}$ and respectively $T_{1}$. Then $T_{1}^{-1} T_{0}$ is a unitary operator on $\boldsymbol{E}_{+}$, so its spectrum consists of complex numbers of norm 1 .

Proposition 4.1. For any $L_{0}, L_{1} \in \mathbf{L a g}$ we have

$$
\operatorname{spec} D_{L_{0}, L_{1}}=\frac{1}{2 \boldsymbol{i}} \exp ^{-1}\left(\operatorname{spec}\left(T_{1}^{-1} T_{0}\right)\right) \text {. }
$$

In particular, the spectrum of $D_{L_{0}, L_{1}}$ consists of finitely many arithmetic progressions with ratio $\pi$ so that the eta invariant of $D_{L_{0}, L_{1}}$ is well defined.

Proof. First observe that any $u \in L^{2}(I, \widehat{\boldsymbol{E}})$ decomposes as a pair

$$
u=\left(u_{+}, u_{-}\right), u_{ \pm} \in L^{2}\left(I, \boldsymbol{E}_{ \pm}\right) .
$$

If $u \in V\left(L_{0}, L_{1}\right)$ is an eigenvector of $D_{L_{0}, L_{1}}$ corresponding to an eigenvalue $\lambda$, then $u$ satisfies the boundary value problems

$$
\begin{gathered}
-i \frac{d u_{+}}{d t}=\lambda u_{+}, \quad i \frac{d u}{d t}=\lambda u_{-}, \\
u_{-}(0)=T_{0} u_{+}(0), \quad u_{-}(1)=T_{1} u_{+}(1) .
\end{gathered}
$$


The equalities (4.4a) imply that

$$
u_{+}(1)=e^{i \lambda} u_{+}(0), u_{-}(1)=e^{-i \lambda} u_{-}(0) .
$$

Using (4.4b) we deduce

$$
e^{i \lambda} T_{1} u_{+}(0)=u_{-}(1)=e^{-i \lambda} u_{-}(0)=e^{-i \lambda} T_{0} u_{+}(0) .
$$

Hence

$$
e^{2 \boldsymbol{i} \lambda} \in \operatorname{spec}\left(T_{1}^{-1} T_{0}\right) \Longrightarrow \lambda \in \frac{1}{2 \boldsymbol{i}} \exp ^{-1}\left(\operatorname{spec}\left(T_{1}^{-1} T_{0}\right)\right) .
$$

Running the above argument in reverse we deduce that any

$$
\lambda \in \frac{1}{2 i} \exp ^{-1}\left(\operatorname{spec}\left(T_{1}^{-1} T_{0}\right)\right)
$$

is an eigenvalue of $D_{L_{0}, L_{1}}$.

We let $\xi\left(L_{0}, L_{1}\right)$ denote the reduced eta invariant of $D_{L_{0}, L_{1}}$,

$$
\xi\left(L_{0}, L_{1}\right)=\frac{1}{2}\left(\operatorname{dim} \operatorname{ker} D_{L_{0}, L_{1}}+\eta_{D_{L_{0}, L_{1}}}(0)\right) .
$$

If $e^{i \theta_{1}}, \ldots, e^{i \theta_{n}}, \theta_{1}, \ldots, \theta_{n} \in[0,2 \pi)$, are the eigenvalues of $T_{1}^{-1} T_{0}$, then the spectrum of $D_{L_{0}, L_{1}}$ is

$$
\operatorname{spec}\left(D\left(L_{0}, L_{1}\right)\right)=\bigcup_{k=1}^{n}\left\{\frac{\theta_{k}}{2}+\pi \mathbb{Z}\right\},
$$

and we deduce as in Section 1 using (1.8) that

$$
\eta_{D_{L_{0}, L_{1}}}=\sum_{\theta_{k} \in(0,2 \pi)}\left(1-\frac{\theta_{k}}{\pi}\right)
$$

and

$$
\xi\left(L_{0}, L_{1}\right)=\frac{1}{2} \sum_{\theta_{k} \in(0,2 \pi)}\left(1-\frac{\theta_{k}}{\pi}\right)+\frac{1}{2} \operatorname{dim} \operatorname{ker} D_{L_{0}, L_{1}} .
$$

On the other hand,

$$
\begin{aligned}
& \frac{1}{2 \pi i} \operatorname{tr} \log \left(-T_{1}^{-1} T_{0}\right)=\frac{1}{2 \pi} \sum_{\theta_{k} \in[0,2 \pi)}\left(\theta_{k}-\pi\right) \\
= & -\frac{1}{2} \sum_{\theta_{k} \in(0,2 \pi)}\left(1-\frac{\theta_{k}}{\pi}\right)-\frac{1}{2} \operatorname{dim} \operatorname{ker}\left(T_{0}-T_{1}\right) .
\end{aligned}
$$

Since $\operatorname{ker} D_{L_{0}, L_{1}} \cong \operatorname{ker}\left(T_{0}-T_{1}\right) \cong L_{0} \cap L_{1}$ we conclude that

$$
\tau\left(T_{0},-T_{1}\right)=\tau\left(-T_{0}, T_{1}\right)=\tau\left(J L_{0}, L_{1}\right)=-\xi\left(L_{0}, L_{1}\right) .
$$

Following [11] (see also [4]) we associate to each triplet of Lagrangians $L_{0}, L_{1}, L_{2}$ the quantity

$$
\omega\left(L_{0}, L_{1}, L_{2}\right):=\tau\left(L_{1}, L_{0}\right)+\tau\left(L_{2}, L_{1}\right)+\tau\left(L_{0}, L_{2}\right),
$$

and we will refer to it as the (Hermitian) Kashiwara-Wall index (or simply the index) of the triplet. Observe that $\omega$ is indeed an integer since (4.1a) implies that

$$
e^{2 \pi i \omega\left(L_{0}, L_{1}, L_{2}\right)}=1 \text {. }
$$

We set

$$
d\left(L_{0}, L_{1}, L_{2}\right):=\operatorname{dim}\left(J L_{0} \cap L_{1}\right)+\operatorname{dim}\left(J L_{1} \cap L_{2}\right)+\operatorname{dim}\left(J L_{2} \cap L_{0}\right) .
$$


Using (4.2) we deduce that for any permutation $\varphi$ of $\{0,1,2\}$ with signature $\epsilon(\varphi) \in$ $\{ \pm 1\}$ we have

$$
\omega\left(L_{0}, L_{1}, L_{2}\right)-\epsilon(\varphi) \omega\left(L_{\varphi(0)}, L_{\varphi(1)}, L_{\varphi(2)}\right)=-d\left(L_{0}, L_{1}, L_{2}\right) \times \begin{cases}0, & \varphi \text { even } \\ 1, & \varphi \text { odd }\end{cases}
$$

We want to apply the above facts to a special choice of $\widehat{\boldsymbol{E}}$. Let $\boldsymbol{I}$ denote the disjoint union of the intervals $I_{1}, \ldots, I_{4}$ introduced in Section 3 . They were obtained by removing the points $\bar{a}_{0}, p_{0}$ and $\bar{b}_{0}$ from the critical level set $C_{0}$; see Figure 2 We interpret $\boldsymbol{I}$ as an oriented 1-dimensional space with boundary and we let

$$
\widehat{\boldsymbol{E}}:=L^{2}(\partial \boldsymbol{I}), \quad \boldsymbol{E}_{ \pm}=L^{2}\left(\partial_{ \pm} \boldsymbol{I}\right) .
$$

The spaces $\boldsymbol{E}_{ \pm}$have canonical bases, and thus we can identify both of them with the standard Hermitian space $\boldsymbol{E}=\mathbb{C}^{4}$. Define $J: \widehat{\boldsymbol{E}} \rightarrow \widehat{\boldsymbol{E}}$ as before. We have a canonical differential operator

$$
D_{0}: C^{\infty}(\boldsymbol{I}, \mathbb{C}) \rightarrow C^{\infty}(\boldsymbol{I}, \mathbb{C}), \quad D_{0}\left[\begin{array}{c}
u_{1} \\
\vdots \\
u_{4}
\end{array}\right]=\left[\begin{array}{c}
-\boldsymbol{i} \frac{d u_{1}}{d t}+\left.\frac{1}{2} h_{0}\right|_{I_{1}} \\
\vdots \\
\vdots \\
-\boldsymbol{i} \frac{d u_{1}}{d t}+\left.\frac{1}{2} h_{0}\right|_{I_{4}}
\end{array}\right] .
$$

We set $\omega_{k}:=\frac{1}{4 \pi} \int_{I_{k}} h_{0} d s$ so that

$$
\omega_{0}=\omega_{1}+\cdots+\omega_{4}, \quad \omega_{0}^{a}=\omega_{1}+\omega_{4}, \omega_{0}^{b}=\omega_{2}+\omega_{3} .
$$

We have a natural restriction map $\gamma: C^{\infty}(\boldsymbol{I}, \mathbb{C}) \rightarrow L^{2}(\partial \boldsymbol{I}, \mathbb{C})=\widehat{\boldsymbol{E}}$, and we define the Cauchy data space of $D_{0}$ to be the subspace

$$
\Lambda_{0}:=\gamma\left(\operatorname{ker} D_{0}\right) \subset \widehat{\boldsymbol{E}} .
$$

We can easily verify that $\Lambda_{0}$ is a Lagrangian subspace of $\widehat{\boldsymbol{E}}$ that is described by the isometry $\boldsymbol{T}_{0}: \boldsymbol{E}_{+} \rightarrow \boldsymbol{E}_{-}$given by the diagonal matrix

$$
\boldsymbol{T}_{0}=\operatorname{Diag}\left(e^{2 \pi i \omega_{1}}, \ldots, e^{2 \pi i \omega_{4}}\right) .
$$

In the remainder of this section we assume that the operators $\mathcal{D}_{0}^{ \pm}$that appear in Theorem 3.1 are invertible.

Proposition 4.2. Let $\mathcal{D}_{0}^{ \pm}$be the operators that appear in Theorem 3.1. Then

$$
\xi_{\mathcal{D}_{0}^{ \pm}}=-\xi\left(\Gamma_{\boldsymbol{T}_{ \pm}}, \Lambda_{0}\right)=\xi\left(\Lambda_{0}, \Gamma_{\boldsymbol{T}_{ \pm}}\right)=-\tau\left(J \Lambda_{0}, \Gamma_{\boldsymbol{T}_{ \pm}}\right) .
$$

Proof. We need to find the spectra of $\boldsymbol{T}_{0}^{-1} \boldsymbol{T}_{ \pm}$. We set $z_{k}=e^{-2 \pi i \omega_{k}}, k=1, \ldots, 4$, $\rho_{+}=e^{i \theta_{+} / 2}$ and $\rho_{-}=e^{-i \theta_{-} / 2}$. Then

$$
\boldsymbol{T}_{0}^{*} \boldsymbol{T}_{+}=\left[\begin{array}{cccc}
0 & 0 & 0 & z_{1} \\
z_{2} \rho_{+} & 0 & 0 & 0 \\
0 & z_{3} & 0 & 0 \\
0 & 0 & z_{4} \rho_{+} & 0
\end{array}\right], \boldsymbol{T}_{0}^{*} \boldsymbol{T}_{-}=\left[\begin{array}{cccc}
0 & 0 & 0 & z_{1} \\
0 & 0 & z_{2} \rho_{-} & 0 \\
0 & z_{3} & 0 & 0 \\
z_{4} \rho_{-} & 0 & 0 & 0
\end{array}\right] .
$$

The eigenvalues of $\boldsymbol{T}_{0}^{*} \boldsymbol{T}_{+}$are the fourth order roots of $\zeta_{+}=\rho_{+}^{2} z_{1} \cdots z_{4}=e^{i\left(\theta_{+}-2 \pi \omega_{0}\right)}$. Hence

$$
\exp ^{-1}\left(\operatorname{spec}\left(\boldsymbol{T}_{0}^{*} \boldsymbol{T}_{+}\right)\right)=\frac{\boldsymbol{i}\left(\theta_{+}-2 \pi \omega_{0}\right)}{4}+\frac{\pi \boldsymbol{i}}{2} \mathbb{Z} .
$$

\footnotetext{
${ }^{9}$ This assumption is satisfied for a generic choice of metric on $\Sigma$.
} 
Using (4.3) we deduce

$$
\operatorname{spec}\left(D_{\Gamma_{\boldsymbol{T}_{+}}, \Lambda_{0}}\right)=\frac{\pi}{4}\left\{\left(\frac{\theta_{+}}{2 \pi}-\omega_{0}\right)+\mathbb{Z}\right\} .
$$

The eigenvalues of $\boldsymbol{T}_{0}^{*} \boldsymbol{T}_{-}$are the square roots of $z_{1} z_{4} \rho_{-}=e^{-\boldsymbol{i}\left(\theta_{-} / 2+2 \pi \omega_{0}^{a}\right)}$ and $z_{2} z_{3} \rho_{-}=e^{-\boldsymbol{i}\left(\theta_{-} / 2+2 \pi \omega_{0}^{b}\right)}$. Hence

$$
\operatorname{spec}\left(D_{\Gamma_{T_{-}}, \Lambda_{0}}\right)=\left\{-\frac{\pi}{2}\left(\frac{\theta_{-}}{4 \pi}+\omega_{0}^{a}\right)+\frac{\pi}{2} \mathbb{Z}\right\} \cup\left\{-\frac{\pi}{2}\left(\frac{\theta_{-}}{4 \pi}+\omega_{0}^{b}\right)+\frac{\pi}{2} \mathbb{Z}\right\} .
$$

The desired conclusion follows using (1.10), (3.8), (3.10) and (4.5).

Theorem 4.3. Under the same assumptions and notation as in Theorem 3.1 we have

$$
\begin{gathered}
i_{A P S}(\bar{\partial})+\lim _{\varepsilon \rightarrow 0^{+}} S F\left(\mathcal{D}_{t} ; \varepsilon<t \leq 1\right)+\lim _{\varepsilon \rightarrow 0^{+}} S F\left(\mathcal{D}_{t},-1 \leq t<-\varepsilon\right) \\
=-\omega\left(J \Lambda_{0}, \Gamma_{\boldsymbol{T}_{+}}, \Gamma_{\boldsymbol{T}_{-}}\right) .
\end{gathered}
$$

Proof. We have

$$
\begin{gathered}
i_{A P S}(\bar{\partial})+\lim _{\varepsilon \rightarrow 0^{+}} S F\left(\mathcal{D}_{t} ; \varepsilon<t \leq 1\right)+\lim _{\varepsilon \rightarrow 0^{+}} S F\left(\mathcal{D}_{t},-1 \leq t<-\varepsilon\right) \\
\stackrel{3.5}{=}-\left(\xi_{+}-\xi\right) \stackrel{4.7}{=}-\tau\left(\Gamma_{\boldsymbol{T}_{+}}, J \Lambda_{0}\right)-\tau\left(J \Lambda_{0}, \Gamma_{\boldsymbol{T}_{-}}\right) \\
=-\omega\left(J \Lambda_{0}, \Gamma_{\boldsymbol{T}_{+}}, \Gamma_{\boldsymbol{T}_{-}}\right)+\tau\left(\Gamma_{\boldsymbol{T}_{-}}, \Gamma_{\boldsymbol{T}_{+}}\right) .
\end{gathered}
$$

To compute $\tau\left(\Gamma_{\boldsymbol{T}_{-}}, \Gamma_{\boldsymbol{T}_{+}}\right)=\tau\left(\boldsymbol{T}_{-}, \boldsymbol{T}_{+}\right)$we need to compute the spectrum of $\boldsymbol{T}_{+}^{*} \boldsymbol{T}_{-}$. A simple computation shows that

$$
\boldsymbol{T}_{+}^{*} \boldsymbol{T}_{-}=\left[\begin{array}{cccc}
0 & 0 & -\boldsymbol{i} & 0 \\
0 & 1 & 0 & 0 \\
-\boldsymbol{i} & 0 & 0 & 0 \\
0 & 0 & 0 & 1
\end{array}\right]
$$

From the second and fourth column we see that 1 is an eigenvalue of $\boldsymbol{T}_{-}^{*} \boldsymbol{T}_{+}$with multiplicity 2 . The other two eigenvalues are $\pm \boldsymbol{i}$, namely the eigenvalues of the $2 \times 2$ minor

This shows that $\tau\left(\boldsymbol{T}_{-}, \boldsymbol{T}_{+}\right)=0$.

$$
\left[\begin{array}{cc}
0 & -i \\
-i & 0
\end{array}\right]
$$

\section{ACKNOWLEDGMENTS}

We would like to thank the anonymous referee for his/her comments.

\section{REFERENCES}

1. V.I. Arnold: The complex Lagrangian Grassmannian, Funct. Anal. and its Appl., 34(2000), 208-210. MR.1802319(2001k:53152)

2. M.F. Atiyah, V.K. Patodi, I.M. Singer: Spectral asymmetry and Riemannian geometry. III, Math. Proc. Camb. Phil. Soc. 79(1976), 71-99. MR0397799 (53:1655c)

3. N. Berline, E. Getzler, M. Vergne: Heat Kernels and Dirac Operators, Springer-Verlag, 1992. MR $1215720(94 \mathrm{e}: 58130)$

4. S. Cappell, R. Lee, E.Y. Miller: On the Maslov index, Comm. Pure Appl. Math. 47(1994), 121-186. MR:1263126 (95f:57045)

5. D.F. Cibotaru: Localization formula in odd K-theory, arXiv: 0901.2563.

6. R. E. Edwards: Functional analysis, Dover Publications, 1995. MR.1320261 (95k:46001) 
7. G.B. Folland: Real Analysis. Modern Techniques and Their Applications, 2nd Edition, John Wiley \& Sons, 1999. MR,1681462 (2000c:00001)

8. P.B. Gilkey: On the index of geometrical operators for Riemannian manifolds with boundary, Adv. Math., 102(1993), 129-183. MR1252030 (95a:58118)

9. G. Grubb: Heat operator trace expansions and index for general Atiyah-Patodi-Singer boundary problems, Comm. Partial Differential Equations, 17(1992), no.11-12, 2031-2077. MR.1194749 (94a:58186)

10. T. Kato: Perturbation Theory for Linear Operators, Springer-Verlag, 1995. MR.1335452 (96a:47025)

11. P. Kirk, M. Lesch: The $\eta$-invariant, Maslov index, and spectral flow for Dirac-type operators on manifolds with boundary, Forum Math., 16(2004), 553-629. MR2044028 (2005b:58029)

12. P. Kronheimer, T. Mrowka: Monopoles and Three-Manifolds, Cambridge University Press, 2007. MR2388043 (2009f:57049)

13. L.I. Nicolaescu: Lectures on the Geometry of Manifolds, 2nd Edition, World Sci. Pub. Co., 2009. MR.1435504 (97k:53001)

14. _ Schubert calculus on the Grassmannian of Hermitian Lagrangian spaces, Adv. in Math. 224(2010), 2361-2434. MR.2652210

15. J. Oprea: Differential Geometry and Its Applications, Mathematical Association of America, distributed by Prentice Hall, Englewood Cliffs, NJ, 2007. MR2327126 (2008k:53002)

16. R. Seely, I.M. Singer: Extending $\bar{\partial}$ to singular Riemann surfaces, J. Geom. Physics, 5(1988), 121-136. MR.1027535 (91f:58101)

17. E.T. Whittaker, G.N. Watson: A Course of Modern Analysis, Cambridge University Press, Fourth Edition, 1927. MR 1424469 (97k:01072)

18. K. Yosida: Functional Analysis, Springer-Verlag, 1980. MR617913 (82i:46002)

19. C.T.C Wall: Non-additivity of the signature, Invent. Math., 7(1969), 269-274. MR0246311 (39:7615)

Instituto de Matemática, Universidade Federal Fluminense, Rua Maria Santos Braga, 24020-140 Niterói, RJ-BrasiL

E-mail address: daniel@mat.uff.br

Department of Mathematics, University of Notre Dame, Notre Dame, Indiana 465564618

E-mail address: nicolaescu.1@nd.edu 